\title{
Article \\ Genotype-Dependent Effect of Silencing of TaCKX1 and TaCKX2 on Phytohormone Crosstalk and Yield-Related Traits in Wheat
}

\author{
Bartosz Jablonski ${ }^{1}$, Andrzej Bajguz ${ }^{2}$, Joanna Bocian ${ }^{1}$, Waclaw Orczyk ${ }^{3}$ iD and Anna Nadolska-Orczyk ${ }^{1, *}$ (D) \\ 1 Department of Functional Genomics, Plant Breeding and Acclimatization Institute-National Research \\ Institute, Radzikow, 05-870 Blonie, Poland; b.jablonski@ihar.edu.pl (B.J.); j.bocian@ihar.edu.pl (J.B.) \\ 2 Laboratory of Plant Biochemistry, Faculty of Biology, University of Bialystok, Ciolkowskiego 1J, \\ 15-245 Bialystok, Poland; abajguz@uwb.edu.pl \\ 3 Department of Genetic Engineering, Plant Breeding and Acclimatization Institute-National Research \\ Institute, Radzikow, 05-870 Blonie, Poland; w.orczyk@ihar.edu.pl \\ * Correspondence: a.orczyk@ihar.edu.pl
}

Citation: Jablonski, B.; Bajguz, A.; Bocian, J.; Orczyk, W.; Nadolska-Orczyk, A. Genotype-Dependent Effect of Silencing of TaCKX1 and TaCKX2 on Phytohormone Crosstalk and Yield-Related Traits in Wheat. Int. J Mol. Sci. 2021, 22, 11494. https:// doi.org/10.3390/ijms222111494

Academic Editors: Fatemeh Maghuly, Eva M. Sehr, Rachit Saxena and David J. Konkin

Received: 5 October 2021

Accepted: 21 October 2021

Published: 25 October 202

Publisher's Note: MDPI stays neutral with regard to jurisdictional claims in published maps and institutional affiliations.

Copyright: (c) 2021 by the authors. Licensee MDPI, Basel, Switzerland. This article is an open access article distributed under the terms and conditions of the Creative Commons Attribution (CC BY) license (https:/ / creativecommons.org/licenses/by/ $4.0 /)$.
Abstract: The influence of silenced TaCKX1 and TaCKX2 on coexpression of other TaCKX gene family members (GFMs), phytohormone regulation and yield-related traits was tested in awned-spike cultivar. We documented a strong feedback mechanism of regulation of TaCKX GFM expression in which silencing of TaCKX1 upregulated expression of TaCKX2 genes and vice versa. Additionally, downregulation of TaCKX2 highly upregulated the expression of TaCKX5 and TaNAC2-5A. In contrast, expression of these genes in silenced TaCKX1 was downregulated. Silenced TaCKX1 $\mathrm{T}_{2}$ lines with expression decreased by $47 \%$ had significantly higher thousand grain weight (TGW) and seedling root mass. Silenced TaCKX2 $\mathrm{T}_{2}$ lines with expression of TaCKX2.2.1 and TaCKX2.2.2 decreased by $33 \%$ and $30 \%$, respectively, had significantly higher chlorophyll content in flag leaves. TaCKX GFM expression, phytohormone metabolism and phenotype were additionally modified by Agrobacteriummediated transformation. Two novel phytohormones, phenylacetic acid (PAA) and topolins, lack of gibberellic acid (GA) and changed phytohormone contents in the 7 days after pollination (DAP) spikes of the awned-spike cultivar compared to a previously tested, awnless one, were detected. We documented that major mechanisms of coregulation of the expression of TaCKX GFMs were similar in different spring wheat cultivars, but, depending on content and composition of phytohormones, regulation of yield-related traits was variously impacted.

Keywords: TaCKX expression; yield traits; wheat genotypes; awned spike; cytokinins; phytohormones

\section{Introduction}

Wheat is globally the third most important cereal crop after maize and rice, and is mainly cultivated in moderate climates. Unfortunately, progress in yield improvement of this crop has failed to meet expectations. Study of gene function, which might play a major role in the creation of yield factors and further modelling of wheat ideotype, is one way for improvement [1].

Cytokinins (CKs) have already been presented as 'a key driver of seed yield' [2]. These hormones occur in plants in more than 30 forms [3] and are distributed spatially throughout the plant and temporally during all developmental stages [4]. There are two types of CKs, isoprenoid and aromatic, the first one being predominant and ubiquitous in higher plants [5,6]. The aromatics are not detected or found at low levels, e.g., 6benzyloamino purine (BA) was found in developing wheat spikes $[7,8]$. Isoprenoid, free base forms, trans-zeatin ( $t Z$ ), cis-zeatin $(c Z)$, isopentenyl adenine (iP) and dihydrozeatin (DZ) as well as their ribosides ( $\mathrm{tZR}, \mathrm{cZR}, \mathrm{iPR}, \mathrm{DZR})$ are active forms. Ribosides are considered to be the major transport forms $[9,10]$. The levels of $\mathrm{CKs}$ are controlled by biosynthesis, destruction, inactivation and translocation [5]. Long-distance translocation of 
root-synthesized cytokinin from xylem to phloem is essential for shoot distribution [11]. Biosynthesis is regulated by isopentenyl transferase genes (IPT), and destruction of $\mathrm{tZ}, \mathrm{cZ}$ and $\mathrm{iP}$, and their ribosides by cytokinin oxidase/dehydrogenase genes (CKX). Inactivation occurs via $\mathrm{O}$ - and $\mathrm{N}$-glucosylation by cytokinin glucosyl transferases (CGTs) to storage forms. A review on IPTs, their function and regulation of stress and yield improvement in plants [4], and reviews on wheat TaCKX and TaCGT gene family members (GFMs) and processes in wheat have been recently reported $[12,13]$. Both processes of inactivation and destruction via CKX enzymes, which are especially important in the regulation of cytokinin level in cereal species, are organ- and species-specific.

The first report on regulation of grain production by CKX GFMs in cereals concerned rice [14], and later barley [15] and wheat [7]. Increased seed yield was also documented in other species including the model Arabidopsis [16] and crop oilseed rape [17]; therefore, it is suggested that cytokinins might 'underpin the second Green revolution' [18]. Moreover, knocking out of $H v C K X 1$ in barley corresponded to greater root length, hair numbers and increased surface area [19], an important component of plant morphology. Knock out mutants of OsCKX11 in rice regulate leaf senescence and grain number by coordination of the source and sink relationship [20]. In all these cited papers, increased seed yield was affected by mutations or RNAi silencing of selected CKX GFMs. Positive results of modification of CK metabolism on yield parameters were also presented in natural variants of TaCKX GFMs. Copy number of TaCKX4 was associated with grain weight and chlorophyll content in flag leaves [21]. Haplotype variants of TaCKX6a02 and TaCKX6-D1 showed a higher filling rate and greater grain size [22,23]. As reviewed, cytokinins are also important components of the response to environmental stresses [24] and mineral nutrient deficiencies [12].

According to newly revised numbering there are $13 \mathrm{TaCKX}$ genes, basically numbered from 1 to 11, and 11 of them are allocated on each diploid genome A, B and D of allohexaploid wheat, giving a total of 35 homologues genes [12]. TaCKX2 underwent gene duplication [25] and their paralogues were primarily subdivided into two subgene families, $T a C K X 2.1$ and TaCKX2.2 [26,27]. However, according to more recent report, TaCKX2.2 is represented by TaCKX2.2.1, TaCKX2.2.2 and TaCKX2.2.3 GFMs [12]. Silencing of TaCKX1 or TaCKX2.2 GFMs coordinated expression of other CKX GFMs, modulated cytokinins and other phytohormone contents in developing spikes and regulated yield-related traits $[7,8]$. A high level of silencing of TaCKX1 strongly downregulated expression of TaCKX11 and upregulated TaCKX2.1, 2.2, 5 and 9 GFMs. This resulted in obtaining a high-yielding phenotype characterized by a higher spike number, grain number, and grain yield, but lower thousand grain weight (TGW) [7]. High silencing of TaCKX2.2.2 was associated with strong upregulation of TaCKX5 and TaCKX11, slight downregulation of TaCKX2.2.1 but the levels of expression of other TaCKX GFMs were comparable to the control [8]. Silenced plants had significantly higher TGW and chlorophyll content in flag leaves, but lower grain number. These changes in coordinated expression of TaCKX1 or TaCKX2.2 with other GFMs resulted in diverse homeostatic balance of cytokinin forms and other phytohormones and final phenotype.

Cytokinins are suggested as signaling molecules of development [10,28]. Therefore, their crosstalk with other phytohormones, e.g., abscisic acid (ABA), gibberellins (GAs) and auxins, is well documented [29]. Both auxins and cytokinins are also necessary in processes of in vitro cereal somatic embryogenesis and plant regeneration [30]. Besides auxins, ABA is considered to have crucial importance for seed development in wheat. Higher cytokinin activity during early wheat kernel development and ABA in later stages corresponded with higher yield [31]. It was also documented in barley that crosstalk among IAA, GAs, $\mathrm{ABA}$ and CKs contributed to regulation of spike and spikelet development, atrophy and abortion [32].

Results of some studies on wheat spike development indicate that the level of expression of TaCKX GFMs and the content of phytohormones are genotypically specific. Profiles of $\mathrm{CK}$ forms and $\mathrm{ABA}$ levels varied greatly among the cultivars, and developmental kernel 
stages and were associated with grain yield potential of wheat cultivars [31]. Moreover, levels of expression of TaCKX GFMs greatly differ among the tested breeding lines and cultivars growing in a controlled environment as well as in the field [33]. Different coexpression groups of TaCKX GFMs, which were formed among modern varieties grown in a controlled environment and in the field, differently regulated yield-related traits [33].

In this paper new data on TaCKX1 and TaCKX2 silencing in the awned-spike cultivar Ostka are compared with already published data on the same GFM silencing in another awnless cultivar, Kontesa $[7,8]$. We found that the phenotypic result of gene silencing is predominantly dependent on the genotype of wheat. Both genotypes differ in phytohormone content and metabolism, which might influence coexpression of the silent gene with other, yield-regulating genes and consequently phytohormone homeostasis and yield-related traits. Some phytohormones, such as PAA and topolins, were newly detected and are suggested to be specific to awned-spike cultivar.

\section{Results}

\subsection{The Levels of Silencing of TaCKX1 and TaCKX2 in $T_{1}$}

The range of relative expression of TaCKX1 in silenced $\mathrm{T}_{1}$ (related to the control $=1.00$ ) was from 0.52 to 0.74 with a mean of 0.66 . The vector dedicated to silencing of TaCKX2.2.23D contained high homology of fragments of a coding sequence to silenced TaCKX2.2.1 and TaCKX2.1 [8]; therefore expression of all of them was measured. The range of relative expression of TaCKX2.2.2 in silenced $\mathrm{T}_{1}$ was from 0.51 to 0.78 and for TaCKX2.2.1 it was from 0.62 to 0.79 . Individual $\mathrm{T}_{1}$ plants with the highest levels of silencing were selected.

\subsection{Coexpression of Silenced Genes with Other TaCKX GFMs and TaNAC2-5A (NAC)}

The range of relative expression of $\mathrm{TaCKX} 1 \mathrm{in}$ selected $\mathrm{T}_{2}$ lines (related to the control $=1.00)$ was from 0.37 to 0.68 with a mean $0.53( \pm 0.03)$ (Figure 1, Table S1). These values in the group of nonsilenced plants segregated in $\mathrm{T}_{2}$ ranged from 0.95 to 1.42 , and the mean was $1.10( \pm 0.05)$. Means of relative expression of TaCKX11, TaCKX5, TaCKX9 and NAC were also decreased compared to the control, and they were $0.65( \pm 0.05), 0.67(0.18), 0.75$ $( \pm 0.06)$ and $0.92( \pm 0.04)$, respectively. These values in the case of TaCKX11 and TaCKX5 were close to TaCKX1 but were significantly higher for TaCKX9 and NAC. Relative values of expression of $\mathrm{TaCKX} 2.2 .1, \mathrm{TaCKX} 2.1$ were more than three times higher than in the case of the silenced gene, reaching $1.75( \pm 0.11)$ and $1.57( \pm 0.09)$, respectively.

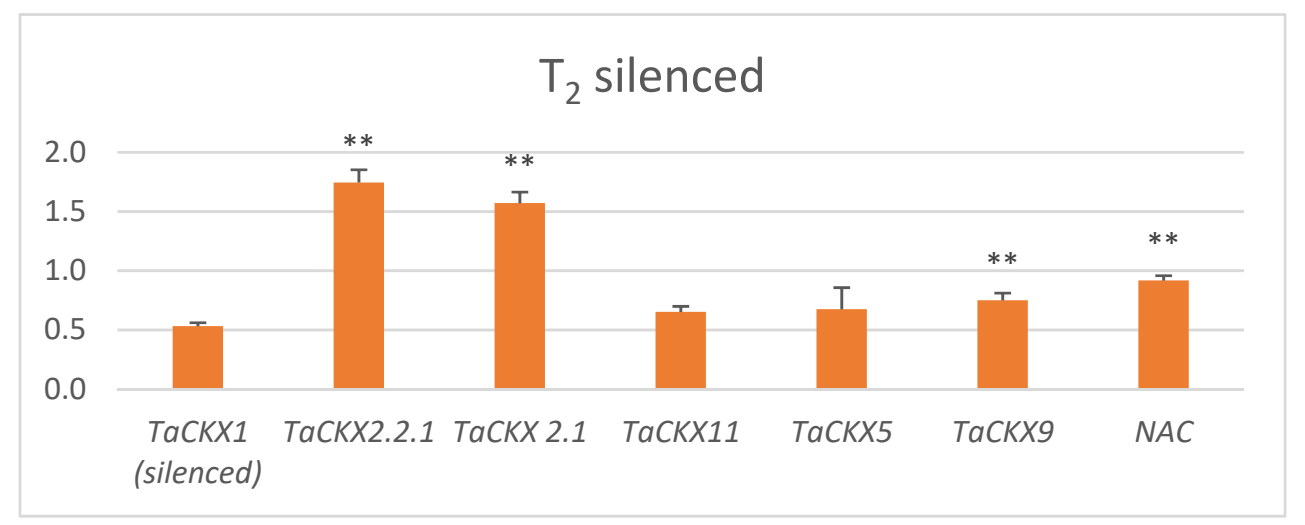

Figure 1. Relative values of expression of TaCKX1 and other TaCKX GFMs, and NAC in silenced $\mathrm{TaCKX} 1$ lines of $\mathrm{T}_{2}$. The error bars denote \pm SE. Significantly different from silenced gene: ${ }^{* *}$ significant at $p \leq 0.01 ; \mathrm{N} 12$ (N-number of objects/tested lines). 
The most silenced in the group of $T a C K X 2$-silenced plants was TaCKX2.2.1, reaching 0.67 ( \pm 0.02$)$ (Figure 2, Table S2). In the case of nonsilenced lines, the range of expression for this gene was from 0.87 to 1.51 and the mean was $1.18( \pm 0.14)$. This value was significantly higher compared with silenced lines. A decreased level of expression compared with the control in the group of silenced plants was also observed for TaCKX2.2.2 $(0.70 \pm 0.04)$ and TaCKX9 $(0.81 \pm 0.10)$. Their values of expression in the group of nonsilenced plants were closer to the control, reaching $1.03(0.07)$ and $0.85( \pm 0.13)$, respectively. The same values for $\mathrm{TaCKX} 2.1$ in both groups were close to the control in silenced lines $(0.99 \pm 0.06)$ and lower in nonsilenced lines $(0.69 \pm 0.03)$, for TaCKX11 slightly higher than the control $(1.12 \pm 0.15$; $1.19 \pm 0.33)$. In the case of $T a C K X 1$ and $N A C$, values of relative expression in both groups were about two times higher than silenced TaCKX2.2.1, reaching $2.29( \pm 0.32), 1.88( \pm 0.22)$ and $1.87( \pm 0.37), 2.12( \pm 0.48)$, respectively. These values differ significantly from the relative expression of silenced TaCKX2.2.1. The level of relative expression of TaCKX5 in the group of silenced plants was more than 13 times higher $(8.80 \pm 1.75)$ and almost 10 times higher $(6.53 \pm 2.47)$ than in the most-silenced TaCKX2.2.1. There were significant differences in expression of TaCKX2.2.1, TaCKX2.2.2 and TaCKX2.1 between nonsilenced and silenced lines. Additionally, the relative value of expression of silenced TaCKX2.2.1 significantly differed from nonsilenced TaCKX2.2.2, and nonsilenced and silenced TaCKX1, TaCKX5, and NAC.

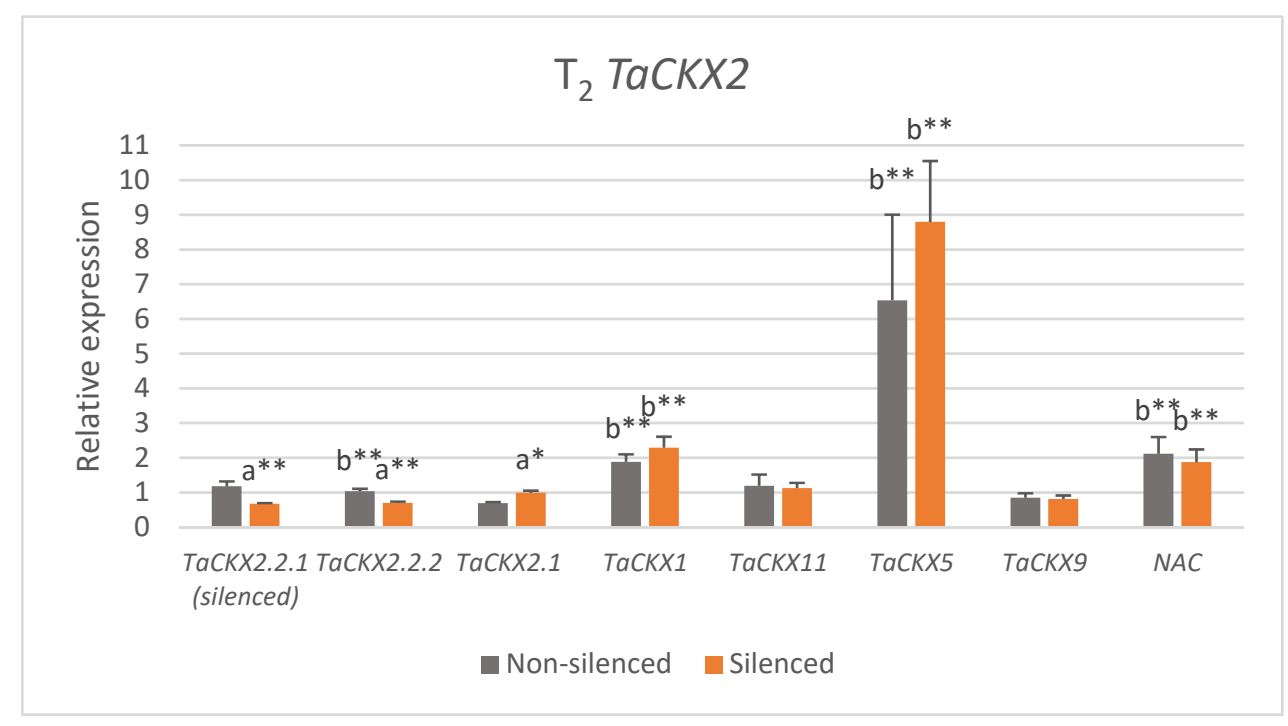

Figure 2. Relative values of expression of TaCKX2 and other TaCKX GFMs, and NAC in silenced and nonsilenced TaCKX2.2.1 lines of $\mathrm{T}_{2}$. The error bars denote \pm SE. Different letters mean significant differences: $\mathrm{a}$ - between silenced and nonsilenced lines; $\mathrm{b}$ - between silenced TaCKX2.2.1 and others. * significant at $p \leq 0.05 ;{ }^{* *}$ significant at $p \leq 0.01$.

\subsection{Phytohormone Contents in Control, Nonsilenced and Silenced Lines}

The highest contents of phytohormones measured in 7 DAP spikes of Ostka, which ranged from 1.6 to above $5 \mathrm{ng} / \mathrm{g}$ biomass, were detected for $\mathrm{tZ}$, tZR, DZOG, PAA and ABA in both silenced and nonsilenced plants of TaCKX1 and TaCKX2 as well as in control lines (Figures $3 \mathrm{~A}$ and $4 \mathrm{~A}$ ). Contents of $\mathrm{cZ}$ and DZOGR were above $1 \mathrm{ng} / \mathrm{g}$ biomass, but contents of most O-glucosides and the others were below $1 \mathrm{ng} / \mathrm{g}$ biomass. 


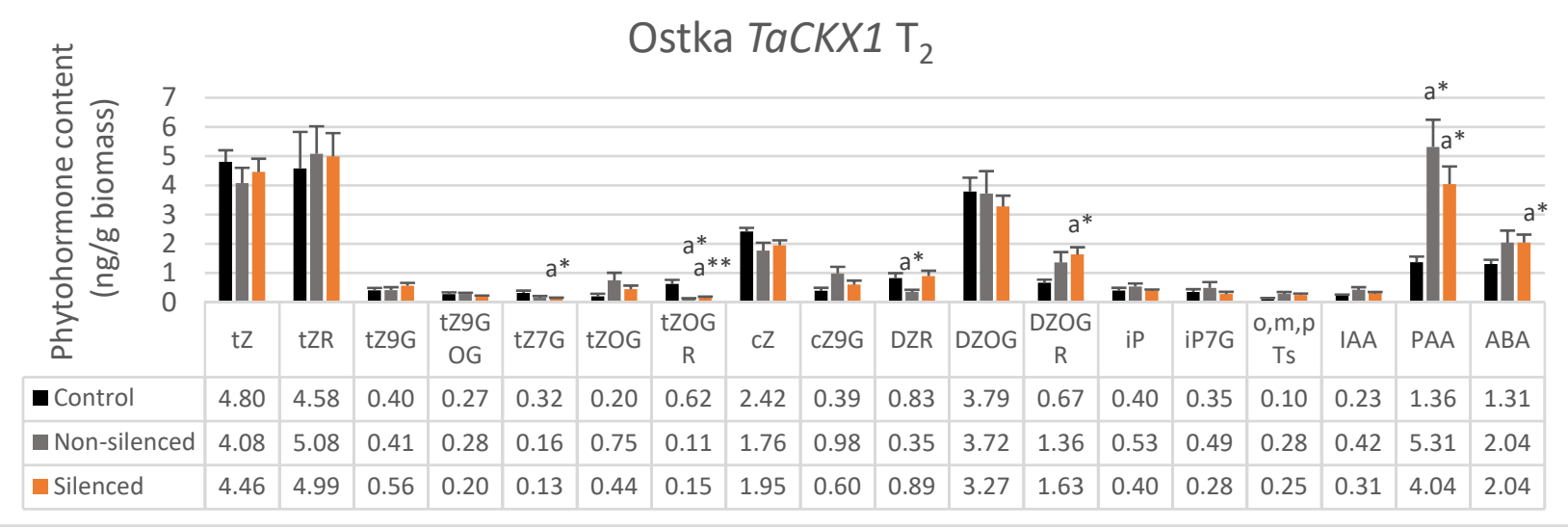

(A)

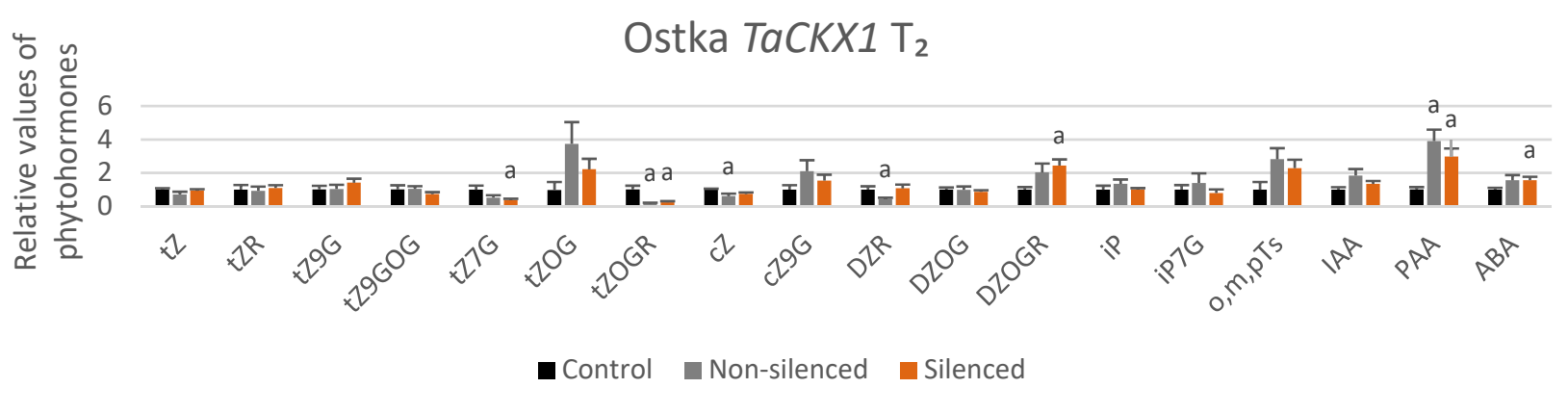

(B)

Figure 3. Phytohormone content (A) and their relative values (B) in control (black bars), nonsilenced (grey bars) and silenced (orange bars) TaCKX1, $\mathrm{T}_{2}$ lines. Trace amounts ( $<0.2 \mathrm{ng} / \mathrm{g}$ biomass): cZOGR, iPR, o,pTs, BA. Not detected: cZOG, cZR, DZ, DZ9G, DZ7G, IBA, IPA, GA. Error bars denote \pm SE. Significant differences between control and nonsilenced or control and silenced lines (a): * significant at $p \leq 0.05 ;{ }^{* *}$ significant at $p \leq 0.01$. No significant difference between nonsilenced and silenced lines.

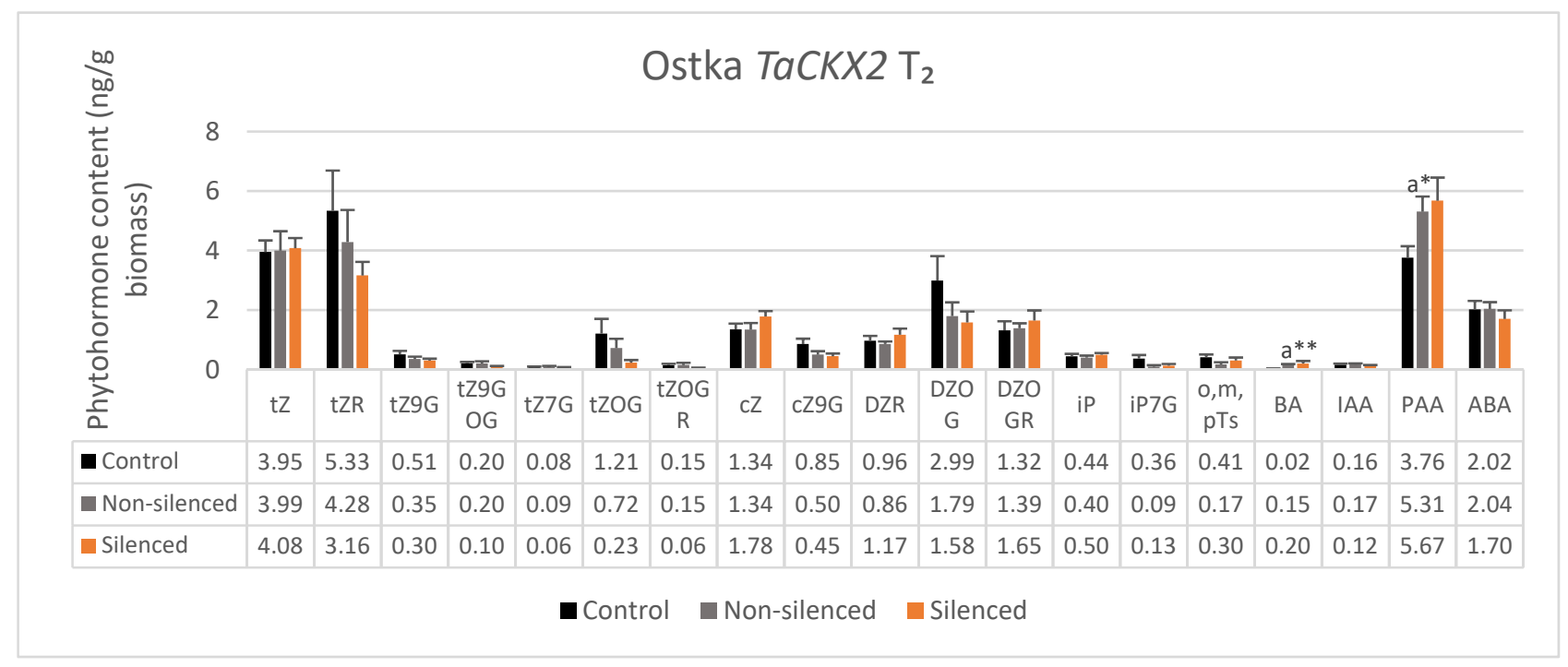

(A)

Figure 4. Cont. 


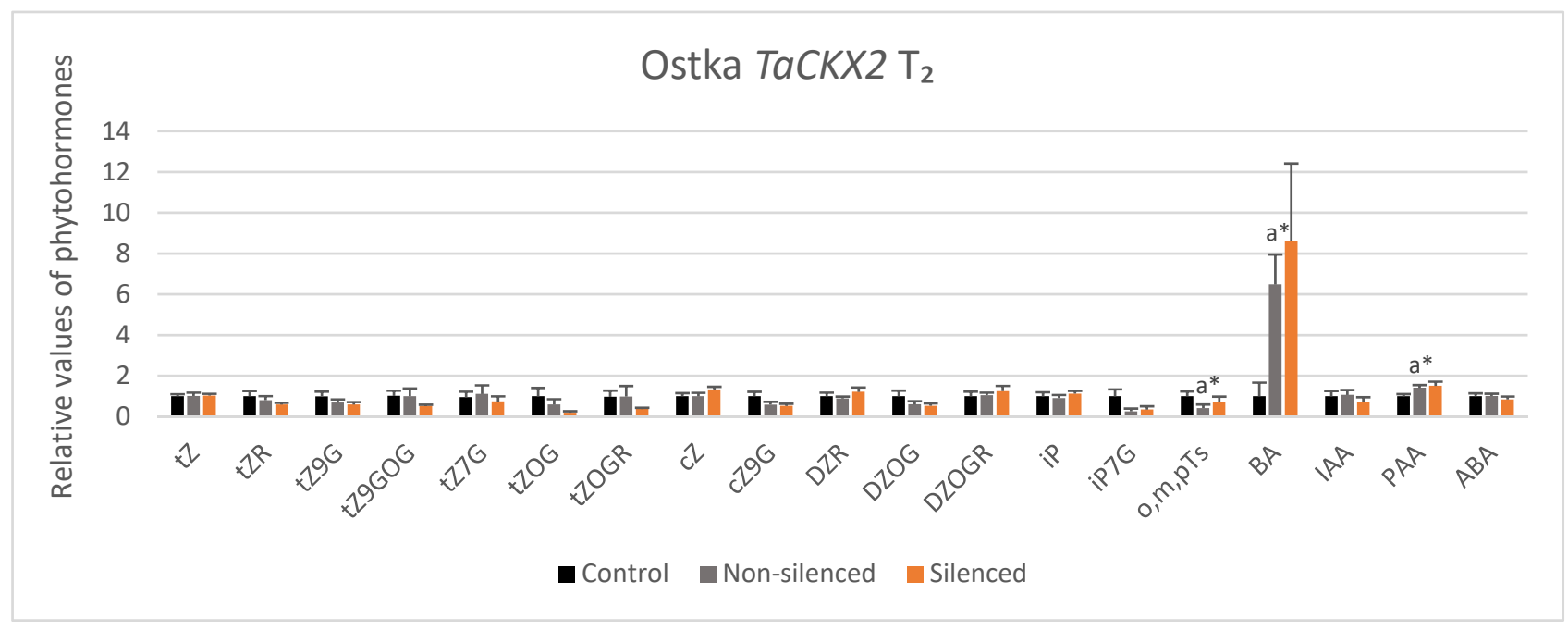

(B)

Figure 4. Phytohormone content (A) and their relative values (B) in control (black bars), nonsilenced (grey bars) and silenced (orange bars) TaCKX2, $\mathrm{T}_{2}$ lines. Trace amounts (<0.1 ng/g biomass): tZ7G, cZR, iPR, oT. Not detected: cZOG, cZOGR, DZ, DZ9G, DZ7G, IPA, IBA, GA. Error bars denote \pm SE. Significant differences between control and nonsilenced or silenced lines (a): * significant at $p \leq 0.05 ;{ }^{* *}$ significant at $p \leq 0.01$.

Besides significant differences in expression level of TaCKX1 between nonsilenced and silenced plants of Ostka, there were no significant differences in contents of active, free base cytokinins, $\mathrm{tZ}, \mathrm{cZ}, \mathrm{iP}$ and other phytohormones between the same groups of lines. The only significant differences were between control and nonsilenced or control and silenced lines (Figure 3A). Already low contents of tZ7G, tZOGR and DZR were significantly lower in one or both groups of TaCKX1-silenced plants than in the controls. The content of DZOGR was significantly higher (more than 2 times) in silenced than in control lines. Already high content of PAA was three to four times higher in nonsilenced and silenced than in control lines. Similarly, content of ABA was significantly higher in silenced plants compared with the control. DZ and their N-glucosides were not detectable, and BA was only detected in silenced plants. Small amounts of meta-topolin $(\mathrm{mT})$ and trace amounts of ortho- and para-topolins (o,pTs) are presented together as o,m,pTs.

Relative values of phytohormone content (related to the control $=1.00$ ) are presented to show differences among values in control, nonsilenced and silenced plants of TaCKX1 lines (Figure 3B). The values of tZOG, cZ9G, DZOGR, o,m,pTs and PAA increased two to almost four times over control in both nonsilenced and silenced lines. A smaller increase was found in the case of IAA and ABA. Relative values of tZ7G, tZOGR, cZ, DZR were significantly lower in one or both groups than in the control.

In the case of TaCKX2 silencing, a significant difference was detected between nonsilenced and silenced plants of Ostka in expression level of TaCKX2.2.1 and TaCKX2.2.2. However, there were no significant differences in contents of active, free base cytokinins, $t Z$, $c Z$, iP and other phytohormones between the same groups of lines (Figure 4A). Significant differences were only noted for BA and PAA between control and nonsilenced lines. Relative values of BA (Figure 4B) were several times higher in nonsilenced and silenced plants compared to the control. Moreover, PAA was significantly higher and o,m,pTs significantly lower in nonsilenced compared with the control lines.

\subsection{Yield-Related Traits in Control, Nonsilenced and Silenced Lines of TaCKX1 and TaCKX2 GFMs}

Silenced TaCKX1 lines had significantly higher TGW than nonsilenced and control lines as well as root mass compared to nonsilenced lines (Figure 5A). Other yield-related traits were not significantly changed; however, grain yield, grain number and root mass were noticeably higher than in nonsilenced plants. 
Relative expression of both TaCKX2.2.1 and TaCKX2.2.2 genes of silenced lines was significantly lower compared to nonsilenced ones (Figure 5B). The only significant difference between silenced and nonsilenced lines in the case of yield-related traits was higher chlorophyll content. Root mass was significantly lower in nonsilenced compared to the control. Grain number and grain yield were slightly lower in silenced compared to nonsilenced lines.

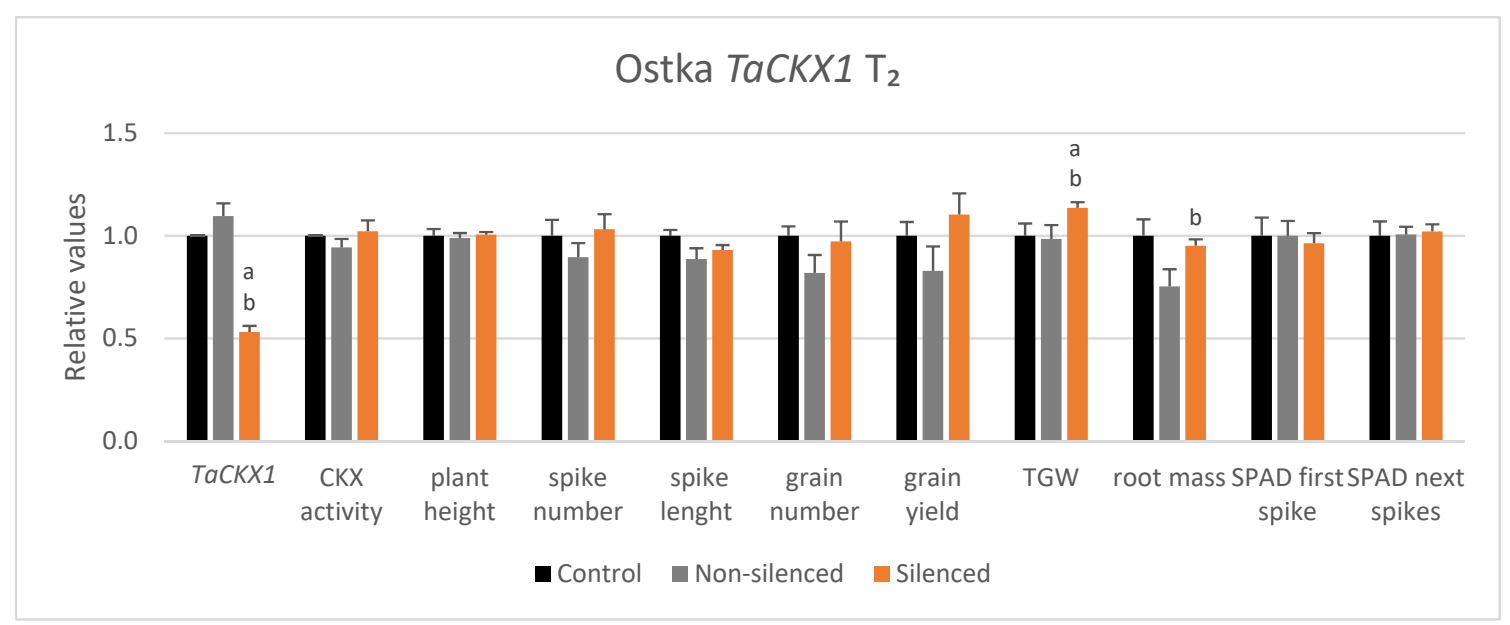

(A)

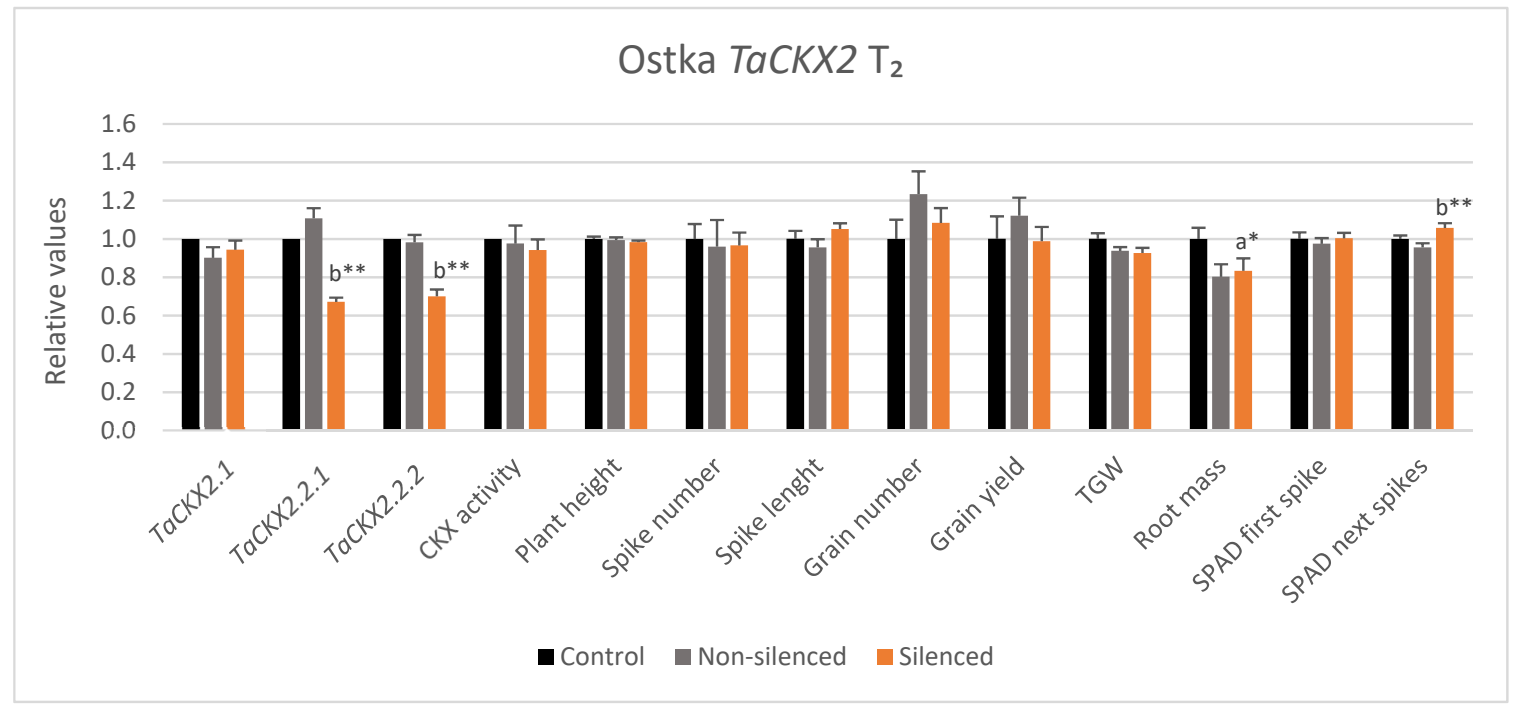

(B)

Figure 5. Relative values of TaCKX1 expression and yield-related traits in TaCKX1 (A) and relative values of TaCKX2.1, 2.2.1 and 2.2.2 expression and yield-related traits in TaCKX2 (B) silenced (orange bars), nonsilenced (grey bars) and control (black bars) of $\mathrm{T}_{2}$ lines. Error bars denote \pm SE. Significant differences between control and silenced (a) and nonsilenced and silenced (b): * significant at $p \leq 0.05 ;{ }^{* *}$ significant at $p \leq 0.01$.

\subsection{Coordinated Effect of TaCKX1 and TaCKX2 Silencing on Expression of Other Genes, Phytohormone Content and Yield-Related Traits}

Silenced TaCKX1 $\mathrm{T}_{2}$ lines showed a $47 \%$ decreased expression (relative to the control) and $52 \%$ decreased ratio indicator (RI silenced per nonsilenced). This level of silencing was combined with significant silencing of TaCKX11, TaCKX5 and TaCKX9 and significantly increased expression of TaCKX2.2.1 and TaCKX2.1 (Figure 6). Silent TaCKX1 $\mathrm{T}_{2}$ lines showed significantly increased TGW and root mass and increased, although not significantly, grain yield and grain number. Ratio indicators for these traits were 1.15, 1.26, 1.33 and 1.19, respectively. Other traits were not changed. The levels of phytohormones were not 
significantly changed. One of the most increased was DZR, with RI 2.56. The most decreased were: iP7G, tZOG, cZ9G with RI 0.57, 0.59 and 0.61, respectively. Interestingly, RI for auxins, IAA and PAA were decreased by $27 \%$ and $24 \%$ and ABA was not changed.

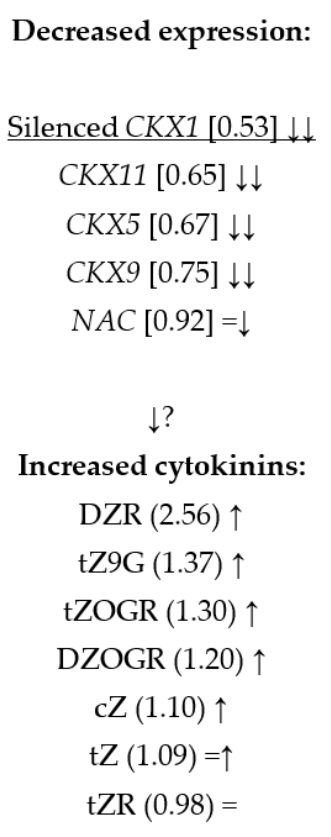

\section{Decreased expression:}
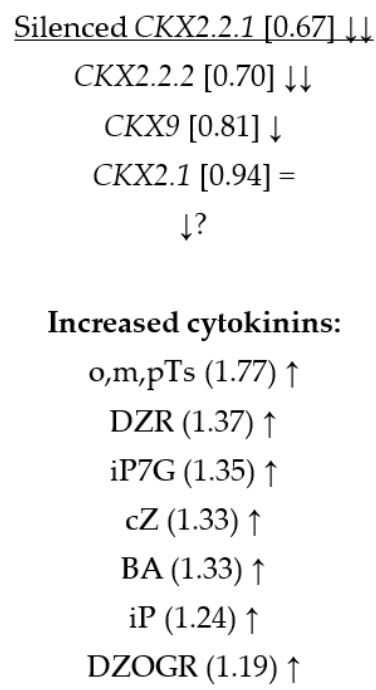$$
\mathrm{tZ}(1.02)=
$$

\section{Increased traits:}

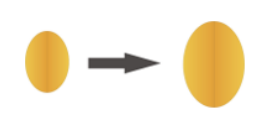

TGW (1.15) $\uparrow \uparrow$

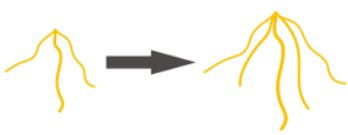

Root mass (1.26) $\uparrow \uparrow$

Grain yield (1.33) $\uparrow$

Grain number (1.19) $\uparrow$

Spike number (1.09) $\uparrow$

CKX act. (1.08) $\uparrow$

Not changed traits:

Spike length $(1.05)=$

Plant height $(1.02)=$ SPAD1 \& ns $(0.96 ; 1.02)=$

(A)

Increased traits:

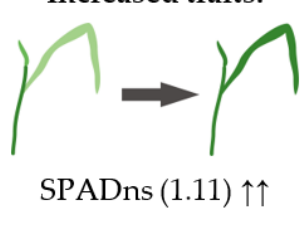

Spike length (1.10) $\uparrow$

Root mass $(1.04)=$

$\operatorname{SPAD} 1(1.03)=$

Decreased traits:

Grain number (0.88) $\downarrow$

Grain yield $(0.88) \downarrow$

Spike number (0.91) $\downarrow$

Not changed traits:

CKX act. $(0.97)=$

Plant height $(0.99)=$ $\operatorname{TGW}(0.99)=$

\author{
Increased expression: \\ CKX2.2.1 [1.75] $\uparrow$ \\ CKX2.1 [1.57] $\uparrow \uparrow$ \\ $\downarrow$ ? \\ Decreased cytokinins: \\ iP7G (0.57) \\ tZOG (0.59) $\downarrow$

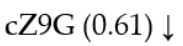 \\ iP $(0.76) \downarrow$

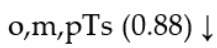 \\ tZ9GOG $(0.70) \downarrow$ \\ tZ7G (0.78) \\ DZOG $(0.88) \downarrow$ \\ Decreased other: \\ IAA $(0.73) \downarrow$ \\ $\operatorname{PAA}(0.76) \downarrow$ \\ $\operatorname{ABA}(1.00)=$
}
Increased expression:
CKX5 [8.80] $\uparrow \uparrow$
CKX1 [2.29] $\uparrow$
NAC [1.87] $\uparrow$
CKX11 [1.12] =
$\downarrow ?$

Decreased cytokinins:

tZOG (0.32)

tZOGR $(0.38) \downarrow$

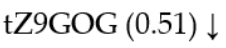

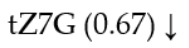

tZR $(0.74) \downarrow$

tZ9G (0.86) $\downarrow$

DZOG $(0.88) \downarrow$

cZ9G (0.90) $\downarrow$

Decreased other:

IAA $(0.69) \downarrow$

$\operatorname{PAA}(1.07)=$

$\operatorname{ABA}(0.84) \downarrow$

(B)

Figure 6. Schematic presentation of effect of $T a C K X 1$ (A) and TaCKX2 (B) silencing on coregulation of expression of other TaCKX GFMs and phytohormone contents and yield-related traits. [ ... ] relative expression to the control; ( . . ) RI silenced or nonsilenced; $\uparrow \uparrow, \downarrow \downarrow$ significantly increased, decreased; $\uparrow, \downarrow$ increased, decreased nonsignificantly.

Silenced TaCKX2 $\mathrm{T}_{2}$ lines showed significantly decreased expression for TaCKX2.2.1 (by 33\%) and expression for TaCKX2.2.2 (by 30\%); however, TaCKX2.1 was not silenced (relative expression $=0.94$ ). These changes of expression were related to significantly 
increased chlorophyll content in flag leaves of second and next spikes. RI of spike length was increased by $10 \%$, but not significantly. Grain number, grain yield and spike number were slightly decreased. Phytohormone contents in silenced TaCKX2 lines were not changed significantly. The highest RI, reaching 1.77 , was for $\mathrm{o}, \mathrm{m}, \mathrm{pTs}$. The most decreased cytokinins were: tZOG, tZOGR, tZ9GOG and tZ7G, with RIs of $0.32,0.38,0.51$ and 0.67 , respectively. Moreover, IAA was decreased by $31 \%$ and ABA by $16 \%$ and PAA content was similar, or slightly increased, compared to that in nonsilenced plants (1.07).

\subsection{Correlations between Yield-Related Traits, Expression of TaCKX GFMs and NAC, and Phytohormones}

There are strong differences in correlations of all tested yield-related traits with growth regulators between control and nonsilenced groups of lines (Tables 1, 2, S3A,B and S4A,B). Although not changed in silenced TaCKX1 lines, plant height strongly negatively correlated with decreasing content of tZOG in the control and with tZOGR in nonsilenced lines. Moreover, there was also a strong positive correlation with $\mathrm{tZ9G}$ and a strong negative correlation with IAA in nonsilenced lines. In contrast, the same trait in silenced lines was positively correlated with PAA. The 33\% increase in grain yield and $19 \%$ increase in grain number in silenced lines (compared to nonsilenced lines) significantly positively correlated with tZ9G in silenced lines but negatively with expression of TaCKX1 as well as DZR, IAA, and in the case of grain number with PAA in nonsilenced lines. Significantly increased in silenced lines (by 15\%), TGW negatively correlated with TaCKX2.1 in silenced lines and strongly negatively with $T a C K X 1$ as well as tZ7G, tZOGR, DZR and $\mathrm{tZ}$ in nonsilenced lines. Root mass, which is the second most significantly increased (by 26\%) trait in silent TaCKX1 lines, correlated negatively with iP in silenced lines and negatively with $\mathrm{TaCKX} 1$ expression, and strongly negatively with $\mathrm{CZ}$, iP7G, tZ, tZ9GOG, tZOG, and PAA in nonsilenced lines. Interestingly, CKX activity positively correlated with PAA in silenced lines and strongly positively with cZ9G in nonsilenced lines.

Table 1. Regulation of yield-related traits by TaCKX GFMs and phytohormones in groups of silenced TaCKX1, nonsilenced and control plants based on correlation coefficients.

\begin{tabular}{|c|c|c|c|}
\hline Control (N6) & Nonsilenced (N6) & $\begin{array}{c}\text { Yield-Related Trait } \\
\text { (RI Silenced/Nonsilenced) }\end{array}$ & Silenced (N12) \\
\hline $\mathrm{tZOG} \downarrow-0.91$ & $\begin{array}{c}\mathrm{tZOGR} \downarrow-0.83 \\
\mathrm{tZ9G} \downarrow 0.85 \\
\mathrm{IAA} \uparrow-0.86 \\
\mathrm{DZR} \downarrow-0.79 \\
\mathrm{DZOG} \uparrow-0.70\end{array}$ & Plant height $(1.02)=$ & $\mathrm{PAA} \downarrow 0.70$ \\
\hline $\begin{array}{c}\mathrm{iP7G} \uparrow-0.74 \\
\mathrm{DZOG} \uparrow-0.84\end{array}$ & $\begin{array}{c}\text { TaCKX } 1 \uparrow \uparrow-0.78 \\
\mathrm{iP7G} \uparrow-0.89 \\
\mathrm{tZ7G} \uparrow-0.78 \\
\mathrm{DZR} \downarrow-0.72 \\
\mathrm{o}, \mathrm{m}, \mathrm{pTs} \uparrow-0.69\end{array}$ & Spike length $(1.05)=$ & TaCKX11 $\downarrow-0.67$ \\
\hline $\begin{array}{c}\mathrm{o}, \mathrm{m}, \mathrm{pTs} \downarrow-0.88 \mathrm{np} \\
\mathrm{tZ9GOG} \uparrow 0.74\end{array}$ & $\begin{array}{c}\mathrm{tZ7G} \uparrow 0.68 \\
\mathrm{tZOG} \uparrow 0.71 \\
\mathrm{DZOGR} \downarrow-0.69 \\
\mathbf{i P \uparrow}-\mathbf{0 . 7 7}\end{array}$ & Spike number (1.15)=个 & nc \\
\hline $\begin{array}{c}\mathrm{cZ} \uparrow 0.89 \\
\mathrm{tZOG} \downarrow-0.77\end{array}$ & $\begin{array}{c}\mathrm{TaCKX} 1 \uparrow \uparrow-0.66 \\
\mathrm{DZR} \downarrow-0.72 \\
\mathrm{iP7G} \uparrow-0.66 \\
\mathrm{PAA} \uparrow-0.67\end{array}$ & Grain number (1.19) $\uparrow$ & tZ9G $\uparrow 0.69$ \\
\hline
\end{tabular}


Table 1. Cont.

\begin{tabular}{|c|c|c|c|}
\hline Control (N6) & Nonsilenced (N6) & $\begin{array}{c}\text { Yield-Related Trait } \\
\text { (RI Silenced/Nonsilenced) }\end{array}$ & Silenced (N12) \\
\hline $\begin{array}{l}\mathbf{t Z} \uparrow-\mathbf{0 . 8 9} \mathrm{np} \\
\mathrm{DZR} \downarrow 0.80 \mathrm{np}\end{array}$ & $\begin{array}{c}\text { TaCKX } 1 \uparrow \uparrow-0.77 \\
\text { tZOGR } \downarrow-0.65 \\
\mathrm{DZR} \downarrow-0.87 \\
\mathrm{iP7G} \uparrow-0.65 \\
\mathrm{IAA} \uparrow-0.71\end{array}$ & Grain yield (1.33) $\uparrow$ & tZ9G $\uparrow 0.73$ \\
\hline $\begin{array}{c}\mathrm{o}, \mathrm{m}, \mathrm{pTs} \downarrow-0.83 \mathrm{np} \\
\mathrm{tZ} \uparrow-\mathbf{0 . 6 8} \\
\mathrm{tZ9GOG} \uparrow 0.71 \\
\mathrm{cZ9G} \downarrow-\mathbf{0 . 7 0}\end{array}$ & 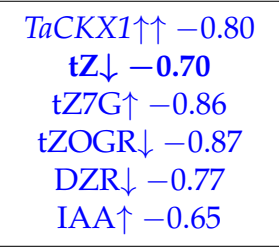 & TGW (1.15) $\uparrow \uparrow$ & $\mathrm{TaCKX} 2.1 \uparrow \uparrow-0.60$ \\
\hline $\begin{array}{c}\mathrm{tZOG} \downarrow 0.64 \\
\mathrm{cZ9G} \downarrow-\mathbf{0 . 6 5}\end{array}$ & $\begin{array}{c}\mathrm{nc} \\
\mathrm{tZ7G \uparrow}-0.68 \\
\mathrm{cZ9G} \downarrow \mathbf{0 . 6 4}\end{array}$ & $\begin{array}{l}\text { SPAD1 (0.96) } \\
\text { SPADns }(1.02\end{array}$ & TaCKX11 $\downarrow 0.58$ \\
\hline $\begin{array}{c}\mathrm{cZ} \uparrow-\mathbf{0 . 8 4} \\
\mathrm{tZOG} \downarrow 0.75 \\
\mathrm{tZOGR} \uparrow-0.70 \\
\mathrm{o}, \mathrm{m}, \mathrm{pTs} \downarrow 0.77 \mathrm{np}\end{array}$ & 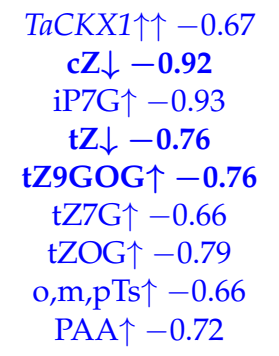 & Root mass (1.26) $\uparrow \uparrow$ & $\mathrm{iP} \downarrow-0.70$ \\
\hline no date & cZ9G个 0.93 & CKX act. $(1.08)=\uparrow$ & $\mathrm{PAA} \downarrow 0.74$ \\
\hline
\end{tabular}

Positive (red) and negative (blue) correlation; nc—not correlated; bold—-degraded by CKX; ( . . ) ratio indicator; for N12 significant from 0.58 and for N6 significant from 0.81 , (N—number of objects/tested lines); $\uparrow, \downarrow$-increase or decrease; $\uparrow \uparrow, \downarrow \downarrow-$ significant increase or significant decrease; $\mathrm{np}$-nonparametric analysis.

Table 2. Regulation of yield-related traits by TaCKX GFMs and phytohormones in groups of silenced TaCKX2.2.1, nonsilenced and control plants based on correlation coefficients.

\begin{tabular}{|c|c|c|c|}
\hline Control (N9) & Nonsilenced (N10) & $\begin{array}{c}\text { Yield-Related Trait } \\
\text { (RI Silenced/Nonsilenced) }\end{array}$ & $\begin{array}{c}\text { Silenced (N9) } \\
\text { (Relative Expression of } \\
\text { TaCKX2.2.1 0.51) }\end{array}$ \\
\hline nc & nc & Plant height $(0.99)=$ & $\begin{array}{c}\text { TaCKX2.2.1 } \downarrow \downarrow-0.88 \\
\text { iP7G } 0.80\end{array}$ \\
\hline nc & $\begin{array}{l}\text { TaCKX2.2.1-0.67 } \\
\text { IAA } \uparrow-0.72\end{array}$ & Spike length $(1.10)=\uparrow$ & 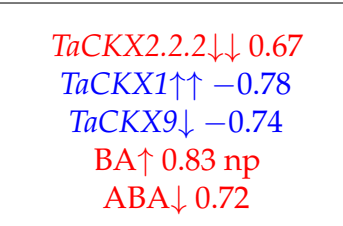 \\
\hline $\mathrm{tZOGR} \uparrow-0.77$ & $\begin{array}{c}\mathrm{tZ} \downarrow 0.77 \mathrm{np} \\
\mathrm{DZOG} \uparrow 0.76 \mathrm{np} \\
\mathrm{iP} \downarrow 0.67 \\
\mathrm{IAA} \uparrow 0.72\end{array}$ & Spike number $(0.91) \downarrow$ & $\begin{array}{l}\mathrm{DZOG} \downarrow 0.76 \\
\mathrm{iP7G \uparrow} 0.75 \mathrm{np}\end{array}$ \\
\hline
\end{tabular}


Table 2. Cont.

\begin{tabular}{|c|c|c|c|}
\hline Control (N9) & Nonsilenced (N10) & $\begin{array}{c}\text { Yield-Related Trait } \\
\text { (RI Silenced/Nonsilenced) }\end{array}$ & $\begin{array}{c}\text { Silenced (N9) } \\
\text { (Relative Expression of } \\
\text { TaCKX2.2.1 0.51) }\end{array}$ \\
\hline nc & $\mathrm{tZ7G \uparrow}-0.78$ & Grain numbe & $\begin{array}{c}\mathrm{tZOGR} \downarrow-0.67 \\
\mathrm{BA} \uparrow 0.74 \mathrm{np}\end{array}$ \\
\hline \multirow[t]{2}{*}{ nc } & $\mathrm{tZ7G} \uparrow-0.78$ & Grain yield $(0.88) \downarrow$ & $\mathrm{BA} \uparrow 0.73 \mathrm{np}$ \\
\hline & nc & & nc \\
\hline nc & $\begin{array}{c}\mathrm{nc} \\
\mathrm{tZ7G} \uparrow-0.69 \mathrm{np} \\
\mathrm{cZ9G} \uparrow 0.78 \\
\mathrm{BA} \downarrow-0.66\end{array}$ & $\begin{array}{c}\text { SPAD1 (1.03) }= \\
\text { SPADns (1.11) } \uparrow\end{array}$ & $\begin{array}{c}\text { nc } \\
\text { TaCKX2.2.2 } \downarrow \downarrow-0.75 \\
\text { DZR } \uparrow 0.80\end{array}$ \\
\hline \multirow[t]{2}{*}{$\mathrm{DZOG} \uparrow 0.72$} & nc & Root mass $(1.04)=$ & cZ9G $\downarrow-0.67 \mathrm{np}$ \\
\hline & $\mathrm{nc}$ & CKX act. $(0.97)=\downarrow$ & nc \\
\hline
\end{tabular}

Positive (red) and negative (blue) correlation; nc—not correlated; bold-degraded by CKX; ( . . ) ratio indicator; for N9 significant from 0.67 , ( $\mathrm{N}$-number of objects, or tested lines); $\uparrow, \downarrow$-increase or decrease; $\uparrow \uparrow, \downarrow \downarrow-$ significant increase or significant decrease; npnonparametric analysis.

Nonsilenced TaCKX1 is negatively correlated with TGW, grain number, grain yield and root mass in nonsilenced lines (Table S3). However, significantly increased expression of TaCKX2.1 in silenced TaCKX1 was negatively correlated with TGW. TaCKX11 negatively correlated with spike length and positively with chlorophyll content in the 1st spike of silenced lines.

Yield-related traits in TaCKX2.2.1-silenced lines were correlated with other TaCKX GFMs and phytohormones. Plant height was negatively correlated with TaCKX2.2.1 and positively with iP7G in silenced lines. Spike length positively correlated with TaCKX2.2.2 and negatively with $T a C K X 1$ and $T a C K X 9$ in silenced lines, but negatively with TaCKX2.2.1 in nonsilenced lines. This trait correlated positively with BA and ABA in silenced lines but negatively with IAA in nonsilenced lines. Grain number and grain yield correlated positively with BA in silenced but negatively with $\mathrm{ZZ7G}$ in nonsilenced lines. There was no correlation between TGW, chlorophyll content measured by SPAD in flag leaf of 1st spike (SPAD first spike/SPAD1) and CKX activity and TaCKX GFMs as well as phytohormones. Chlorophyll content in flag leaves of the next spikes (SPAD next spikes/SPADns) negatively correlated with $\mathrm{TaCKX}$ 2.2.2 and positively correlated with DZR in silenced lines but negatively correlated with $\mathrm{tZ7G}$, BA and positively correlated with cZ9G in nonsilenced lines. Root mass was negatively correlated with cZ9G in silenced lines. 


\subsection{Differences in Composition and Contents of Phytohormones in 7 DAP Spikes of Ostka vs. Kontesa}

Two new auxins, PAA and o,m,p topolins and lack of GA were detected in 7 DAP spikes of Ostka compared to earlier data concerning 7 DAP spikes of Kontesa [7,8]. Moreover, some phytohormone contents in 7 DAP spikes of Ostka (Figures $3 \mathrm{~A}$ and $4 \mathrm{~A}$ ) and Kontesa were significantly different. These data are visualized in Figures 7, S1 and S2. In TaCKX1-silenced plants, the content of tZR was 44 and 8 times higher in nonsilenced Ostka compared to nonsilenced Kontesa and silenced Ostka compared to silenced Kontesa, respectively. In TaCKX2-silenced plants, these differences were only slightly smaller and were 13 and 5 times, respectively. In contrast, the content of tZ9G in TaCKX1-silenced plants was 12 and 5 times lower in nonsilenced Ostka compared to nonsilenced Kontesa and silenced Ostka compared to silenced Kontesa, respectively, and in TaCKX2-silenced plants these differences were 5 and 10 times smaller.

Big differences between cultivars were also observed in contents of DZOG and iP. Both groups of silenced and nonsilenced lines of Ostka, independently of the silenced gene, contained about six to seven times more DZOG and two to four times more iP, although the content of the latter was below $0.5 \mathrm{ng} / \mathrm{g}$ biomass.
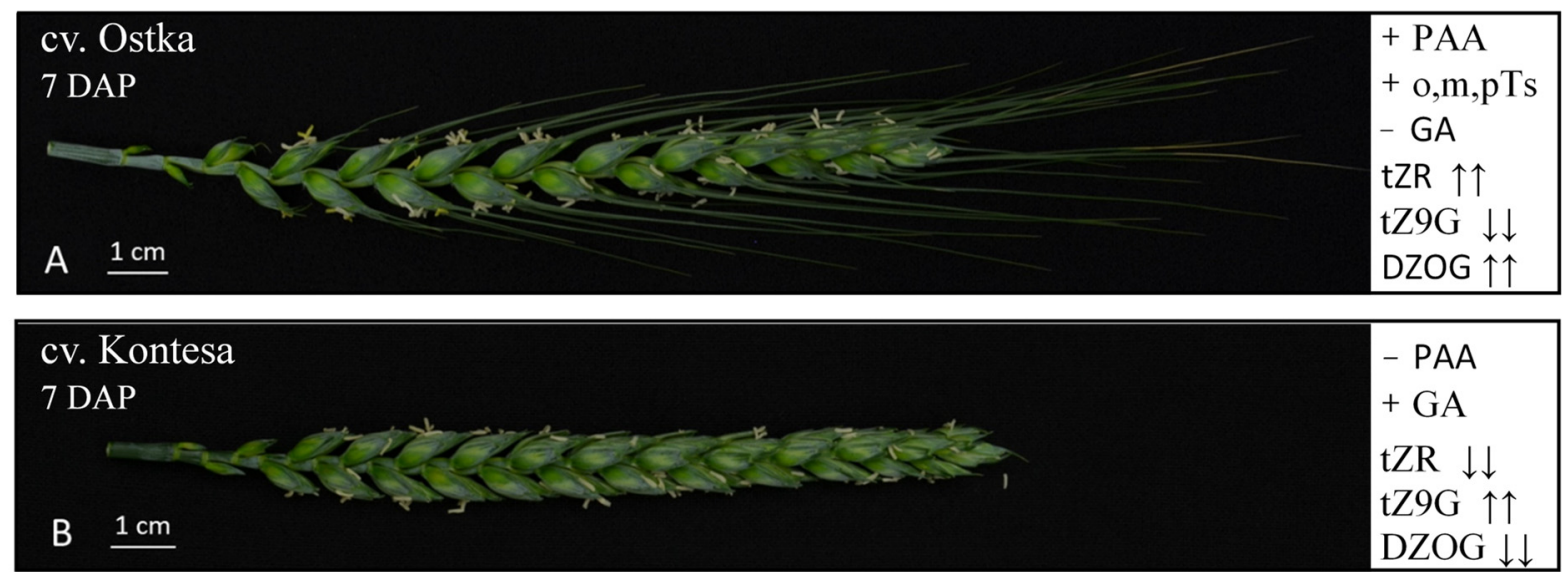

Figure 7. Comparison of composition and contents of phytohormones in 7 DAP spikes of Ostka and Kontesa. + newly detected; - not found; $\uparrow$ strongly increased; $\downarrow \downarrow$ strongly decreased.

\section{Discussion}

The object of our research is the middle part of the 7 DAP spike of wheat. This is the early developmental stage, named the cellularization stage, which lasts up to 10 days [34], and is the middle of the cell division or cell expansion stage [35,36] or the late milk stage [31]. This stage was characterized by a high level of expression of tested TaCKX1 and TaCKX2 genes in wheat [27] and their orthologs in barley [37], and their silencing at this stage corresponded to yield-related traits $[7,8,15,38]$.

\subsection{Feedback Mechanism of Regulation of TaCKX GFM Expression and Its Coordination}

Silencing of TaCKX1 in the cultivar Ostka coordinated significantly increased expression of TaCKX2.2.1 and TaCKX2.1 genes, and silencing of TaCKX2 genes caused a significant increase in expression of TaCKX1. A similar, but not so strong, feedback mechanism of regulation of expression was observed in the case of silencing of the same TaCKX1 and $T a C K X 2$ genes in the cultivar Kontesa [7,8]. Moreover, decreased expression of TaCKX1 in Ostka was coordinated with decreased expression of TaCKX11, TaCKX5 and TaCKX9, but silencing of TaCKX2 genes was coregulated with several-times increased TaCKX5 and NAC2, in both silenced and nonsilenced lines. This means that regulation of expression of these two genes was mainly independent of the level of silencing of other TaCKX GFMs; however, it is dependent on coculture with Agrobacterium carrying the TaCKX1 or TaCKX2 silencing cassette (discussed below). This feedback mechanism of regulation of their expression 
resulted in stable CKX enzyme activity and maintained homeostasis of phytohormones. As reported before, most TaCKX GFMs are organ or tissue specific [27,33]. The TaCKX1 and $T a C K X 2$ GFMs are specifically expressed in developing grains. Expression of TaCKX5 and TaCKX9 is specific to younger organs from seedling roots to 0 DAP spikes, but TaCKX11 and NAC2 are expressed in all organs [33]. Levels of relative expression of NAC2 in roots, leaves and 14 DAP spikes reported for wheat by He et al. [39] were comparable. Moreover, orthologous to TaCKX11 of wheat, rice OsCKX11 [12] was, as in wheat, expressed through the plant, but predominantly in roots, leaves and panicles [20]. Coordinated decrease in silenced $\mathrm{TaCKX} 1$ with TaCKX11 was also reported by Jablonski et al. [7] and coordinated increase in TaCKX5 with silenced TaCKX2 GFMs was reported by Jablonski et al. [8]. Therefore, not only expression pattern, as was hypothesized before [37], but also integrated action indicate the role of CKX GFMs in barley and wheat development. We might also conclude that these major mechanisms of coregulation of expression of TaCKX GFMs are similar in different spring wheat cultivars.

\subsection{Agrobacterium-Mediated Transformation Influences TaCKX GFM Expression, Phytohormone Metabolism and Phenotype}

Different types of biotic and abiotic stress impact cytokinin homeostasis and its crosstalk with other phytohormones [24]. We might suppose that one such stress might be genetic modifications, which include in vitro culture and Agrobacterium-mediated transformation with different vectors. Indeed, there were significant differences in TaCKX GFM expression and phytohormone metabolism between in vitro origin, control $\mathrm{F}_{2}$ and nonsilenced $\mathrm{T}_{2}$ lines segregated from silenced ones. For example, in the TaCKX1 silencing experiment, plant height was strongly negatively regulated by tZOGR and IAA and positively regulated by tZ9G in nonsilenced lines but strongly negatively regulated by tZOG in control lines. Grain number and grain yield were exclusively regulated in control lines: strongly positively by $\mathrm{cZ}$ and negatively by $\mathrm{tZ}$ and positively by DZR, respectively. Since both groups were obtained from the same in vitro culture and cultivated during the same time, under the same conditions, the only difference is that nonsilenced lines originated from in vitro culture cocultured with Agrobacterium carrying the respective silencing vector. Therefore, these differences might be explained by epigenetic or genetic changes in expression of plant genes influencing the process of plant transformation and their impact on the next generations.

During Agrobacterium-mediated transformation, transferred DNA (T-DNA) uses the host's plant factors to be integrated to the plant genome and to express transgenes [40,41]. Regulation of T-DNA expression by DNA methylation induces the host-plant defense response by global changes in plant growth regulation. As recently reported, Agrobacterium VirE2, which coats single-stranded T-DNA molecules, might alter the transcriptome and proteome of the Arabidopsis root system to facilitate transformation [42]. Moreover, such modifications of DNA methylation and other epigenetic modifications occur during in vitro culture as well [43].

\subsection{Phytohormonal Content and Composition Are Cultivar-Dependent}

Significant changes in coregulation of expression of TaCKX GFMs in silenced plants do not significantly influence phytohormonal homeostasis in the tested cultivar. However, two novel phytohormones in 7 DAP spikes of the awned-spike Ostka (silenced and nonsilenced) compared to awnless Kontesa previously tested in the same conditions, were found [7,8]. The first one was the auxin PAA, detected in high concentrations, above $3 \mathrm{ng} / \mathrm{g}$ biomass. Others specifically identified in Ostka were aromatic cytokinins, and topolins with a concentration below $1 \mathrm{ng} / \mathrm{g}$ biomass. The naturally occurring auxin PAA was detected at significant levels in different tissues of higher plants, e.g., in shoots of Triticum aestivum [44] and young shoots of oats and barley as well as different tissues including dry seeds, inflorescences and roots of Arabidopsis [45]. It was indicated that this auxin activates a very similar subset of genes as IAA; therefore, both auxins might have overlapping regulatory roles. However, IAA and PAA formed slightly different auxin receptor complexes, and 
showed different transport characteristics, which suggested their different physiological effects $[45,46]$. In whole, mature grains, PAA is one of the components of phenolic acids from dietary fibers [47]. To date there is no evidence of PAA presence in developing grains, and its specialized function remains unknown.

Topolins, similarly to BA, belong to naturally occurring, aromatic CKs found in plants [48-50]. These CKs are products of monohydroxylation of the aromatic ring at the side chain of BA, and depending on the side there are three isomers; $\mathrm{oT}, \mathrm{mT}$ and $\mathrm{pT}$. Knowledge about their role in plants is lacking. However, presence of both topolins and PAA in 7 DAP spikes of Ostka testifies that they are important components of phytohormonal homeostasis in this organ.

Moreover, in contrast to Kontesa, GA was not detected in any group of silenced and nonsilenced lines of Ostka, and contents of several other phytohormones significantly differ between cultivars. The contents of tZR and DZOG were several to several dozen times higher in 7 DAP spikes of Ostka compared to Kontesa. In contrast, the content of tZ9G was several times lower in Ostka. These differences in composition and content of phytohormones indicate differences in phytohormone metabolism and transport between tested cultivars. tZR is a transporter metabolite [10], and its much higher content in 7 DAP spikes of Ostka might indicate more active transport of this riboside from roots to spikes of this cultivar. The presence of several times more DZOG in Ostka than Kontesa suggests a more active process of irreversible inactivation of DZ by O-glucosyltransferase in the former. The higher content of $\mathrm{tZ9G}$ and $\mathrm{tZOG}$ indicates higher activity of degradation of tZR by N-glucosyl transferase or O-glucosyl transferase [49] in Kontesa.

These differences in composition and content of phytohormones in 7 DAP spikes of both wheat cultivars are probably reflected in their different phenotypes. The most visible are awned spikes in Ostka and awnless spikes in Kontesa. As reported, wheat awns play an important role in photosynthesis, grain production, and drought tolerance [51,52], and awnless wheats produce more grains per spike, but their size is reduced compared to awned ones [53]. The authors suggested that allocation of assimilates to rapidly developing awns decreased spikelet number and floret fertility, and in consequence, grain number. Regulation of awn length and grain production by cytokinin metabolism was documented in rice by the $A n-2$ gene. The gene encodes Lonely Guy Like protein 6, which catalyzes the final step of cytokinin synthesis in O. rufipogon [54]. It is feasible that these new compounds of the hormonal pool in 7 DAP spikes of Ostka, PAA and topolins, are the main regulators of awns, which should be further tested.

\subsection{Regulation of Yield-Related Traits Is Gene- and Genotype-Dependent}

Silenced TaCKX1 lines of Ostka with almost 50\% decreased expression are characterized by significantly higher TGW and root mass and higher grain number and grain yield. Indeed, expression of this gene is negatively correlated with these traits in nonsilenced lines. However, decreased expression of TaCKX1 is coexpressed with decreased expression of $\mathrm{TaCKX} 11$ and increased expression of TaCKX2.1 in both compared cultivars, Ostka and Kontesa [7]. Furthermore, TGW in silenced Ostka was significantly higher, but in Kontesa it was significantly lower compared to nonsilenced lines. Thus, silencing of TaCKX1 coordinates expression of other TaCKX GFMs in different wheat cultivars in a similar way, but regulates TGW in the opposite way. Consequently, regulation of this trait is genotype-dependent. Again, higher TGW in the awned-spike cultivar Ostka is in agreement with previous discussion [53]. Regardless, other traits such as higher grain number, yield and root mass in TaCKX1 silenced lines of both cultivars were regulated in the same manner. A similar effect of $H v C K X 1$ silencing on yield-related traits to Ostka and partly to Kontesa was observed in barley [15]. TaCKX1 is an ortholog of barley HvCKX1, showing $90-92 \%$ homology with three homologous copies of TaCKX1 from A, B, and D genomes. Silenced $\mathrm{T}_{1}$ lines of barley were characterized by higher (like in Ostka) TGW and root mass, and higher (like in both wheat cultivars) seed number and yield. It is possible that this similar mechanism of regulation of TGW between silenced TaCKX1 lines of wheat, 
cultivar Ostka, and silenced HvCKX1 lines of barley, cultivar Golden Promise, is dependent on the similar spike phenotype. While spikes of Kontesa are awnless, spikes of Ostka and Golden Promise are characterized by long awns. Activity of CKX enzyme in barley lines was decreased and correlated with higher root mass. A significant decrease in CKX enzyme activity and changed root morphology with greater root length were observed for barley lines with knockout $H v C K X 1$; however, in these mutants, grain yield was not changed [19]. Based on these data we can conclude that these differences in changes of the level of CKX activity and their influence on phenotype are dependent on the number of homologous copies of TaCKX1 and $\mathrm{Hv} \mathrm{CKX} 1$ and the level or lack of gene expression. Therefore, while both genes play a very important role in yield improvement, their knock-out might not result in the expected phenotype.

In rice, the main role of OsCKX11, orthologous to wheat, and expressed through the plant, was coordination of the source and sink relationship by opposite regulation of leaf senescence and grain number [20]. Increased cytokinin levels in flag leaves of the Ostka mutant downregulated ABA synthesis and upregulated ABA-degrading genes, indicating antagonistic function between cytokinins and $\mathrm{ABA}$. These results are difficult to compare with those of wheat, since expression of TaCKX11 was only partly (by 35\%) decreased and was strongly coordinated with decreased and increased expression of other TaCKX GFMs. Moreover, a noticeable increase in grain number was observed in both species.

Significantly higher chlorophyll content in flag leaves of the second and next spikes of silenced TaCKX2 lines of Ostka is the result of 30\% decreased TaCKX2.2.2 and TaCKX2.2.1. Indeed, this trait was negatively regulated by $\mathrm{TaCKX} 2.2 .2$ expression but positively by DZ riboside, the only isoprenoid cytokinin which is not susceptible to degradation by CKX [5]. In nonsilenced plants the trait was up-regulated by $c Z 9 G$ and down-regulated by $\mathrm{tZ7G}$ and BA. Both $c Z 9 G$ and $t Z 7 G$ are the products of degradation of active $c Z, t Z$ or their ribosides by N-glucosyl-transferase, and act antagonistically in regulation of chlorophyll content. Likewise, both silenced TaCKX2 genes act antagonistically since TaCKX2.2.1 negatively regulates plant height and spike length and $T a C K X 2.2 .2$ positively regulates spike length but negatively regulates chlorophyll content in flag leaves. Silencing of these genes in both wheat cultivars, Ostka and Kontesa [8], is coregulated in the same way, with increased expression of TaCKX5 and TaCKX1. Additionally, silencing of TaCKX2 in Kontesa resulted in a partly different phenotype than in Ostka, characterized by higher TGW, grain number and chlorophyll content [8]. Again, silencing of the selected TaCKX gene in two different spring cultivars or genotypes of wheat similarly coordinated coexpression of other TaCKX GFMs, but variously impacted phenotype. We documented that the effect of silencing of these two GFMs, TaCKX1 and TaCKX2, gave opposite phenotypic results regarding TGW in both cultivars. Therefore, coordinated silencing of both genes, TaCKX1 and TaCKX2, would increase TGW and yield more efficiently, independently of genotype.

As discussed above, leaf senescence in rice was regulated by OsCKX11, which antagonistically coordinated cytokinin levels and ABA [20]. However, expression of its orthologue in silenced $\mathrm{TaCKX} 2$ lines of the wheat cultivar Ostka, characterized by significantly higher chlorophyll content in flag leaves, was not changed and ABA was only slightly decreased. The effect of silencing of TaCKX2 lines of the wheat cultivar Kontesa [8] was more similar to rice and more different to Ostka. Expression of TaCKX11 was increased, ABA content decreased by $50 \%$ and chlorophyll content in flag leaves increased. Moreover, GA level was reduced to zero and ABA level was positively correlated with TaCKX2.2.2 expression and negatively with cZOG. Therefore, the mechanisms involved in leaf senescence of awned and awnless wheats differ.

Cytokinins and ABA are indicated as two major regulators of plant senescence; a high level of CKs might delay senescence, but high ABA content has an opposite effect $[55,56]$. Moreover, $\mathrm{ABA}$ is an important regulator of assimilate transport. Although, the content of $\mathrm{ABA}$ in 7 DAP spikes of both wheat cultivars was rather high (around $2 \mathrm{ng} / \mathrm{g}$ biomass); its negative correlation with chlorophyll content in flag leaves was documented only in TaCKX2 silenced lines of Kontesa [8]. 
Results of regulation of yield-related traits in wheat by the TaCKX6a02 allele selected among random, isogenic lines of wheat [22], recently renamed as TaCKX2.1-3D [12], were contradictory to Ostka but similar to Kontesa. Opposite data were reported for a haplotype variant of TaCKX6-D1 [23], i.e., TaCKX2.2.1-3D. These contradictions suggest the importance of the level of silencing or knockout of the gene of interest in the individual genetic background. Both factors modulate other regulatory genes and take part in maintaining hormonal homeostasis.

Interestingly, higher grain number and grain yield in silenced TaCKX1 lines of Ostka were positively correlated with $\mathrm{tZ9G}$, which is the product of N-glucosyl transferase degradation of active $\mathrm{tZ}$ or tZR [49]. The same product was strongly positively correlated with CKX activity in nonsilenced lines. These correlations indicate a relationship between regulation of both traits by the enzyme $\mathrm{CKX}$, which catalyzes degradation of $\mathrm{tZ9G}$, as was observed in maize and Arabidopsis [49,57]. Similarly, grain yield was also significantly correlated with CKX activity and tZGs in silenced TaCKX1 lines of Kontesa [7].

Significantly higher root mass in silenced TaCKX1 lines of Ostka was downregulated by iP, but in nonsilenced and control lines was mainly downregulated by higher TaCKX1 expression and $\mathrm{cZ}$ content. The same trait in silenced TaCKX1 lines of Kontesa is downor up-regulated by $\mathrm{TaCKX} 11$ and TaCKX9, which coordinate CZOG metabolism [7]. cZOG is a product of reversible inactivation of $\mathrm{cZ}$ by O-glucosyl transferase [13] and was not detected in 7 DAP spikes of Ostka. However, in both cultivars, seedling root weight is upregulated mainly by $\mathrm{cZ}$, which is metabolized in a different way. In contrast, root mass in silenced TaCKX2 lines of both cultivars, Ostka and Kontesa, was not changed compared with nonsilenced lines, but in Ostka it was downregulated by cZ9G metabolism.

Unchanged plant height in silenced TaCKX1 lines of Ostka was negatively regulated by IAA in nonsilenced and positively by PAA in silenced lines; therefore these two auxins act antagonistically. In silenced TaCKX1 lines of Kontesa, plant height was regulated positively by GA, which is absent in 7 DAP spikes of Ostka, and positively by GA [7]. Therefore, regulation of this trait in these two cultivars is different and mainly depends on compositions and content of phytohormones.

Comparison of data of TaCKX1 and TaCKX2 gene silencing obtained in an awned-spike cultivar to an awnless one previously published shed new light on designing wheat ideotypes for breeding. Despite similarities of major mechanisms of coregulation of expression of tested genes with others from the same gene family in both genotypes, yield-related traits were variously impacted. We found that 7 DAP spikes of the two cultivars differ significantly in composition and content of phytohormones, which is suggested to be the main reason for the differences in the final phenotype. It was also documented that yield-related traits are modified by the process of Agrobacterium-mediated transformation. Moreover, it was confirmed that apart from genotype, phenotype is also dependent on the level of gene expression or silencing. In conclusion, future design of wheat ideotype for breeding should take into consideration selected genotype and requires more complex study than analysis of gene function.

\section{Materials and Methods}

\subsection{Plant Material}

Donor material was the spring wheat cultivar Ostka Smolicka provided by Plant Breeding Company Smolice Ltd., Co.-IHAR-PIB Group, Smolice 146, 63-740 Smolice, Poland. This is a high-yielding, quality (bristly) variety, class A, with high lodging resistance, high frost tolerance and awned spikes. According to our previous experiments this cultivar was susceptible to Agrobacterium-mediated transformation.

\subsection{Vector Construction, Plant Transformation and Selection of Transgenic Lines}

The same hpRNA type of silencing cassettes for TaCKX1 and for TaCKX2 silencing as previously constructed for Kontesa [7,8] were used for transformation of Ostka. Both 
cassettes were cloned into the binary vector pBract207 (Crop Transformation (BRACT) | John Innes Centre (jic.ac.uk), accessed on 25 October 2021) as previously reported.

Growth conditions of donor plants in a growth chamber, Agrobacterium-mediated transformation, and selection of putative transgenic plants were performed as we described for the cultivar Kontesa $[7,8]$.

\subsection{Quantitative RT-qPCR and CKX Activity Assay}

Quantitative RT-qPCR was measured in 7 days after pollination (DAP) spikes of plants selected from $T_{1}$ and $T_{2}$ generations as previously described $[7,8]$. The middle part of the first spike from each experimental plant was collected, frozen in liquid nitrogen, powdered and divided for both analyses, RT-qPCR and CKX activity assay. The RTqPCR was done for seven target genes named according to Chen et al. [12]: TaCKX1 (JN128583), TaCKX2.1 (JF293079), TaCKX2.2.1 (FJ648070), TaCKX2.2.2 (GU084177), TaCKX11 (JN128587), TaCKX5 and TaCKX9 (JN128591), as well as TaNAC2-5A (AY625683). In brackets are accession numbers from NCBI used in previously published papers as reported by Ogonowska et al. [27]. Sequences of specific primers are shown in Supplementary Table S5. ADP-ribosylation factor (Ref2) (AB050957) was used as a reference gene in all qPCR reactions. The relative expression levels in transgenic plants were calculated according to expression in control, in vitro plants, set to 1.00. Transgenic plants were divided into two groups: silenced with the relative expression $\leq 0.79$ and nonsilenced with the relative expression $\geq 0.8$. All measurements were performed in three technical replicates in at least nine biological replicates for one group of lines. Exact numbers of plants and lines tested are included in Supplementary Materials.

CKX activity was analyzed in the second part of the sample (after grounding the sample was divided for two: one for RNA isolation and one for enzyme activity analyzes) from the same spikes as previously described $[15,33]$.

\subsection{Quantification of Cytokinins, Auxins, $A B A$ and $G A 3$}

For quantification of CKs, 27 standards were used: trans-zeatin (tZ), trans-zeatin riboside (tZR), trans-zeatin-9-glucoside (tZ9G), trans-zeatin-7-glucoside (tZ7G), trans-zeatinO-glucoside (tZOG), trans-zeatin riboside-O-glucoside (tZROG), trans-zeatin-9-glucoside-Oglucoside (tZ9GOG), trans-zeatin-9-glucoside riboside (tZ9GR), cis-zeatin (cZ), cis-zeatinriboside (cZR), cis-zeatin O-glucoside (cZOG), cis-zeatin 9-glucoside (cZ9G), cis-zeatin-Oglucoside-riboside (cZROG), dihydrozeatin (DZ), dihydrozeatin-riboside (DZR), dihydrozeatin9-glucoside (DZ9G), dihydrozeatin-7-glucoside (DZ7G), dihydrozeatin-O-glucoside (DZOG), dihydrozeatin riboside-O-glucoside (DZROG), $\mathrm{N}^{6}-\left(\Delta^{2}\right.$-isopentenyl)adenine (iP), $\mathrm{N}^{6}$ isopentenyladenosine (iPR), $\mathrm{N}^{6}$-isopentenyladenosine-7-glucoside (iP7G), para-topolin ( $\mathrm{pT}$ ), meta-topolin (mT), ortho-topolin (oT), 6-benzylaminopurine (6-BAP). Standards for quantification of five auxins were: indole-3-acetic acid (IAA), indole-3-butyric acid (IBA), indole-3propionic acid (IPA), 1-naphthaleneacetic acid (NAA), and 2-phenylacetic acid (PAA). Moreover, $\mathrm{ABA}$ and $\mathrm{GA}_{3}$ were quantified. The procedure is described by Jablonski et al. [7].

\subsection{Analysis of Phenotypic Traits and Statistical Analysis}

These analyses were performed as previously reported for Kontesa [8].

Supplementary Materials: The following are available online at https:/ / www.mdpi.com/article / 10.3390 /ijms222111494/s1.

Author Contributions: Conceptualization, A.N.-O. and W.O.; methodology, B.J., J.B. and A.B.; software, J.B.; validation, B.J. and A.N.-O.; formal analysis, B.J. and A.N.-O.; investigation, B.J. and A.B.; data curation, B.J. and A.N.-O.; writing-original draft preparation, A.N.-O. and W.O.; writing-review and editing, A.N.-O.; visualization, A.N.-O., B.J. and J.B.; supervision, A.N.-O.; project administration, A.N.-O.; funding acquisition, A.N.-O. All authors have read and agreed to the published version of the manuscript. 
Funding: This research was funded by the National Science Centre, Poland grant No. UMO-2014/ 13/B/NZ9/02376 and grant No. UMO-2020/ 37/B/NZ9/00744.

Institutional Review Board Statement: Not applicable.

Informed Consent Statement: Not applicable.

Acknowledgments: We thank Malgorzata Wojciechowska, Izabela Skuza and Agnieszka Glowacka for excellent technical assistance.

Conflicts of Interest: The authors declare no conflict of interest.

\section{References}

1. Nadolska-Orczyk, A.; Rajchel, I.K.; Orczyk, W.; Gasparis, S. Major genes determining yield-related traits in wheat and barley. Theor. Appl. Genet. 2017, 130, 1081-1098. [CrossRef]

2. Jameson, P.E.; Song, J.C. Cytokinin: A key driver of seed yield. J. Exp. Bot. 2016, 67, 593-606. [CrossRef]

3. Kisiala, A.; Kambhampati, S.; Stock, N.L.; Aoki, M.; Emery, R.J.N. Quantification of Cytokinins Using High-Resolution AccurateMass Orbitrap Mass Spectrometry and Parallel Reaction Monitoring (PRM). Anal. Chem. 2019, 91, 15049-15056. [CrossRef]

4. Nguyen, H.N.; Lai, N.; Kisiala, A.B.; Emery, R.J.N. Isopentenyltransferases as master regulators of crop performance: Their function, manipulation, and genetic potential for stress adaptation and yield improvement. Plant Biotechnol. J. 2021, 19, 1297-1313. [CrossRef]

5. Sakakibara, H. Cytokinins: Activity, biosynthesis, and translocation. Annu. Rev. Plant Biol. 2006, 57, 431-449. [CrossRef]

6. Mok, D.W.S.; Mok, M.C. Cytokinin metabolism and action. Annu. Rev. Plant. Physiol. Plant Mol. Biol. 2001, 52, 89-118. [CrossRef] [PubMed]

7. Jablonski, B.; Ogonowska, H.; Szala, K.; Bajguz, A.; Orczyk, W.; Nadolska-Orczyk, A. Silencing of TaCKX1 Mediates Expression of Other TaCKX Genes to Increase Yield Parameters in Wheat. Int. J. Mol. Sci. 2020, 21, 4809. [CrossRef] [PubMed]

8. Jablonski, B.; Szala, K.; Przyborowski, M.; Bajguz, A.; Chmur, M.; Gasparis, S.; Orczyk, W.; Nadolska-Orczyk, A. TaCKX2.2 Genes Coordinate Expression of Other TaCKX Family Members, Regulate Phytohormone Content and Yield-Related Traits of Wheat. Int. J. Mol. Sci. 2021, 22, 4142. [CrossRef] [PubMed]

9. Kudo, T.; Kiba, T.; Sakakibara, H. Metabolism and Long-distance Translocation of Cytokinins. J. Integr. Plant Biol. 2010, 52, 53-60. [CrossRef]

10. Sakakibara, H. Cytokinin biosynthesis and transport for systemic nitrogen signaling. Plant J. 2021, 105, 421-430. [CrossRef]

11. Zhao, J.; Ding, B.; Zhu, E.; Deng, X.; Zhang, M.; Zhang, P.; Wang, L.; Dai, Y.; Xiao, S.; Zhang, C.; et al. Phloem unloading via the apoplastic pathway is essential for shoot distribution of root-synthesized cytokinins. Plant Physiol. 2021, 186, 2111-2123. [CrossRef]

12. Chen, L.; Zhao, J.Q.; Song, J.C.; Jameson, P.E. Cytokinin dehydrogenase: A genetic target for yield improvement in wheat. Plant Biotechnol. J. 2020, 18, 614-630. [CrossRef]

13. Chen, L.; Zhao, J.; Song, J.C.; Jameson, P.E. Cytokinin glucosyl transferases, key regulators of cytokinin homeostasis, have potential value for wheat improvement. Plant Biotechnol. J. 2021, 19, 878-896. [CrossRef]

14. Ashikari, M.; Sakakibara, H.; Lin, S.Y.; Yamamoto, T.; Takashi, T.; Nishimura, A.; Angeles, E.R.; Qian, Q.; Kitano, H.; Matsuoka, M. Cytokinin oxidase regulates rice grain production. Science 2005, 309, 741-745. [CrossRef]

15. Zalewski, W.; Galuszka, P.; Gasparis, S.; Orczyk, W.; Nadolska-Orczyk, A. Silencing of the HvCKX1 gene decreases the cytokinin oxidase/dehydrogenase level in barley and leads to higher plant productivity. J. Exp. Bot. 2010, 61, 1839-1851. [CrossRef]

16. Bartrina, I.; Otto, E.; Strnad, M.; Werner, T.; Schmulling, T. Cytokinin Regulates the Activity of Reproductive Meristems, Flower Organ Size, Ovule Formation, and Thus Seed Yield in Arabidopsis thaliana. Plant Cell 2011, 23, 69-80. [CrossRef]

17. Schwarz, I.; Scheirlinck, M.T.; Otto, E.; Bartrina, I.; Schmidt, R.C.; Schmulling, T. Cytokinin regulates the activity of the inflorescence meristem and components of seed yield in oilseed rape. J. Exp. Bot. 2020, 71, 7146-7159. [CrossRef]

18. Jameson, P.E.; Song, J.C. Will cytokinins underpin the second 'Green Revolution'? J. Exp. Bot. 2020, 71, 6872-6875. [CrossRef]

19. Gasparis, S.; Przyborowski, M.; Kala, M.; Nadolska-Orczyk, A. Knockout of the HvCKX1 or HvCKX3 Gene in Barley (Hordeum vulgare L.) by RNA-Guided Cas9 Nuclease Affects the Regulation of Cytokinin Metabolism and Root Morphology. Cells 2019, 8, 782. [CrossRef]

20. Zhang, W.; Peng, K.X.; Cui, F.B.; Wang, D.L.; Zhao, J.Z.; Zhang, Y.J.; Yu, N.N.; Wang, Y.Y.; Zeng, D.L.; Wang, Y.H.; et al. Cytokinin oxidase/dehydrogenase OsCKX11 coordinates source and sink relationship in rice by simultaneous regulation of leaf senescence and grain number. Plant Biotechnol. J. 2020, 19, 335-350. [CrossRef]

21. Chang, C.; Lu, J.; Zhang, H.P.; Ma, C.X.; Sun, G.L. Copy Number Variation of Cytokinin Oxidase Gene Tackx4 Associated with Grain Weight and Chlorophyll Content of Flag Leaf in Common Wheat. PLoS ONE 2015, 10, e0145970. [CrossRef]

22. Lu, J.; Chang, C.; Zhang, H.P.; Wang, S.X.; Sun, G.; Xiao, S.H.; Ma, C.X. Identification of a Novel Allele of TaCKX6a02 Associated with Grain Size, Filling Rate and Weight of Common Wheat. PLoS ONE 2015, 10, e0144765. [CrossRef]

23. Zhang, L.; Zhao, Y.L.; Gao, L.F.; Zhao, G.Y.; Zhou, R.H.; Zhang, B.S.; Jia, J.Z. TaCKX6-D1, the ortholog of rice OsCKX2, is associated with grain weight in hexaploid wheat. New Phytol. 2012, 195, 574-584. [CrossRef] 
24. Cortleven, A.; Leuendorf, J.E.; Frank, M.; Pezzetta, D.; Bolt, S.; Schmulling, T. Cytokinin action in response to abiotic and biotic stresses in plants. Plant Cell Environ. 2019, 42, 998-1018. [CrossRef]

25. Mameaux, S.; Cockram, J.; Thiel, T.; Steuernagel, B.; Stein, N.; Taudien, S.; Jack, P.; Werner, P.; Gray, J.C.; Greenland, A.J.; et al. Molecular, phylogenetic and comparative genomic analysis of the cytokinin oxidase/dehydrogenase gene family in the Poaceae. Plant Biotechnol. J. 2012, 10, 67-82. [CrossRef]

26. Zhang, J.P.; Liu, W.H.; Yang, X.M.; Gao, A.N.; Li, X.Q.; Wu, X.Y.; Li, L.H. Isolation and characterization of two putative cytokinin oxidase genes related to grain number per spike phenotype in wheat. Mol. Biol. Rep. 2011, 38, 2337-2347. [CrossRef]

27. Ogonowska, H.; Barchacka, K.; Gasparis, S.; Jablonski, B.; Orczyk, W.; Dmochowska-Boguta, M.; Nadolska-Orczyk, A. Specificity of expression of TaCKX family genes in developing plants of wheat and their co-operation within and among organs. PLoS ONE 2019, 14, e0214239. [CrossRef]

28. Kieber, J.J.; Schaller, G.E. Cytokinin signaling in plant development. Development 2018, 145, dev149344. [CrossRef]

29. Guo, W.; Chen, L.; Herrera-Estrella, L.; Cao, D.; Tran, L.P. Altering Plant Architecture to Improve Performance and Resistance. Trends Plant Sci. 2020, 25, 1154-1170. [CrossRef]

30. Przetakiewicz, A.; Orczyk, W.; Nadolska-Orczyk, A. The effect of auxin on plant regeneration of wheat, barley and triticale. Plant Cell Tissue Organ Cult. 2003, 73, 245-256. [CrossRef]

31. Nguyen, H.N.; Perry, L.; Kisiala, A.; Olechowski, H.; Emery, R.J.N. Cytokinin activity during early kernel development corresponds positively with yield potential and later stage ABA accumulation in field-grown wheat (Triticum aestivum L.). Planta 2020, 252, 76. [CrossRef] [PubMed]

32. Youssef, H.M.; Hansson, M. Crosstalk among hormones in barley spike contributes to the yield. Plant Cell Rep. 2019, 38, 1013-1016. [CrossRef] [PubMed]

33. Szala, K.; Ogonowska, H.; Lugowska, B.; Zmijewska, B.; Wyszynska, R.; Dmochowska-Boguta, M.; Orczyk, W.; Nadolska-Orczyk, A. Different sets of $\mathrm{TaCKX}$ genes affect yield-related traits in wheat plants grown in a controlled environment and in field conditions. BMC Plant Biol. 2020, 20, 496. [CrossRef] [PubMed]

34. Zhang, S.; Ghatak, A.; Bazargani, M.M.; Bajaj, P.; Varshney, R.K.; Chaturvedi, P.; Jiang, D.; Weckwerth, W. Spatial distribution of proteins and metabolites in developing wheat grain and their differential regulatory response during the grain filling process. Plant J. 2021, 107, 669-687. [CrossRef]

35. Gao, X.P.; Francis, D.; Ormrod, J.C.; Bennett, M.D. Changes in Cell Number and Cell-Division Activity during Endosperm Development in Allohexaploid Wheat, Triticum aestivum L. J. Exp. Bot. 1992, 43, 1603-1609. [CrossRef]

36. Hess, J.R.; Carman, J.G.; Banowetz, G.M. Hormones in wheat kernels during embryony. J. Plant Physiol. 2002, 159, 379-386. [CrossRef]

37. Zalewski, W.; Gasparis, S.; Boczkowska, M.; Rajchel, I.K.; Kala, M.; Orczyk, W.; Nadolska-Orczyk, A. Expression patterns of $\mathrm{HvCKX}$ genes indicate their role in growth and reproductive development of barley. PLoS ONE 2014, 9, e115729. [CrossRef]

38. Zalewski, W.; Orczyk, W.; Gasparis, S.; Nadolska-Orczyk, A. HvCKX2 gene silencing by biolistic or Agrobacterium-mediated transformation in barley leads to different phenotypes. BMC Plant Biol. 2012, 12, 206. [CrossRef]

39. He, X.; Qu, B.Y.; Li, W.J.; Zhao, X.Q.; Teng, W.; Ma, W.Y.; Ren, Y.Z.; Li, B.; Li, Z.S.; Tong, Y.P. The Nitrate-Inducible NAC Transcription Factor TaNAC2-5A Controls Nitrate Response and Increases Wheat Yield. Plant Physiol. 2015, 169, $1991-2005$. [CrossRef]

40. Lacroix, B.; Citovsky, V. The roles of bacterial and host plant factors in Agrobacterium-mediated genetic transformation. Int. J. Dev. Biol. 2013, 57, 467-481. [CrossRef]

41. Gelvin, S.B. Traversing the cell: Agrobacterium T-DNA's journey to the host genome. Front. Plant Sci. 2012, 3, 52. [CrossRef]

42. Lapham, R.A.; Lee, L.Y.; Xhako, E.; Gomez, E.G.; Nivya, V.M.; Gelvin, S.B. Agrobacterium VirE2 Protein Modulates Plant Gene Expression and Mediates Transformation From Its Location Outside the Nucleus. Front. Plant Sci. 2021, 12, 684192. [CrossRef]

43. Miguel, C.; Marum, L. An epigenetic view of plant cells cultured in vitro: Somaclonal variation and beyond. J. Exp. Bot. 2011, 62, 3713-3725. [CrossRef]

44. Korasick, D.A.; Enders, T.A.; Strader, L.C. Auxin biosynthesis and storage forms. J. Exp. Bot. 2013, 64, 2541-2555. [CrossRef]

45. Sugawara, S.; Mashiguchi, K.; Tanaka, K.; Hishiyama, S.; Sakai, T.; Hanada, K.; Kinoshita-Tsujimura, K.; Yu, H.; Dai, X.H.; Takebayashi, Y.; et al. Distinct Characteristics of Indole-3-Acetic Acid and Phenylacetic Acid, Two Common Auxins in Plants. Plant Cell Physiol. 2015, 56, 1641-1654. [CrossRef]

46. Cook, S.D.; Ross, J.J. The auxins, IAA and PAA, are synthesized by similar steps catalyzed by different enzymes. Plant Signal Behav. 2016, 11, e1250993. [CrossRef]

47. Calinoiu, L.F.; Vodnar, D.C. Whole Grains and Phenolic Acids: A Review on Bioactivity, Functionality, Health Benefits and Bioavailability. Nutrients 2018, 10, 1615. [CrossRef]

48. Nedved, D.; Hosek, P.; Klima, P.; Hoyerova, K. Differential Subcellular Distribution of Cytokinins: How Does Membrane Transport Fit into the Big Picture? Int. J. Mol. Sci. 2021, 22, 3428. [CrossRef]

49. Spichal, L. Cytokinins-Recent news and views of evolutionally old molecules. Funct. Plant Biol. 2012, 39, 267-284. [CrossRef]

50. Mok, M.C.; Martin, R.C.; Dobrev, P.I.; Vankova, R.; Ho, P.S.; Yonekura-Sakakibara, K.; Sakakibara, H.; Mok, D.W.S. Topolins and hydroxylated are substrates of cytokinin thidiazuron derivatives O-glucosyltransferase with position specificity related to receptor recognition. Plant Physiol. 2005, 137, 1057-1066. [CrossRef] 
51. Wang, D.; Yu, K.; Jin, D.; Sun, L.; Chu, J.; Wu, W.; Xin, P.; Gregova, E.; Li, X.; Sun, J.; et al. Natural variations in the promoter of Awn Length Inhibitor 1 (ALI-1) are associated with awn elongation and grain length in common wheat. Plant J. 2020, 101, 1075-1090. [CrossRef]

52. Reynolds, M.; Tuberosa, R. Translational research impacting on crop productivity in drought-prone environments. Curr. Opin. Plant. Biol. 2008, 11, 171-179. [CrossRef]

53. Rebetzke, G.J.; Bonnett, D.G.; Reynolds, M.P. Awns reduce grain number to increase grain size and harvestable yield in irrigated and rainfed spring wheat. J. Exp. Bot. 2016, 67, 2573-2586. [CrossRef]

54. Gu, B.G.; Zhou, T.Y.; Luo, J.H.; Liu, H.; Wang, Y.C.; Shangguan, Y.Y.; Zhu, J.J.; Li, Y.; Sang, T.; Wang, Z.X.; et al. An-2 Encodes a Cytokinin Synthesis Enzyme that Regulates Awn Length and Grain Production in Rice. Mol. Plant 2015, 8, 1635-1650. [CrossRef]

55. Nooden, L.D.; Guiamet, J.J.; John, I. Senescence mechanisms. Physiol. Plant. 1997, 101, 746-753. [CrossRef]

56. Haberer, G.; Kieber, J.J. Cytokinins. New insights into a classic phytohormone. Plant Physiol. 2002, 128, 354-362. [CrossRef]

57. Hosek, P.; Hoyerova, K.; Kiran, N.S.; Dobrev, P.I.; Zahajska, L.; Filepova, R.; Motyka, V.; Muller, K.; Kaminek, M. Distinct metabolism of $\mathrm{N}$-glucosides of isopentenyladenine and trans-zeatin determines cytokinin metabolic spectrum in Arabidopsis. New Phytol. 2020, 225, 2423-2438. [CrossRef] 